\title{
The Hippocampal System as the Cortical Resource Manager: a model connecting psychology, anatomy and physiology
}

\author{
L. Andrew Coward
}

Department of Computer Science, Australian National University, Canberra, ACT 0200, Australia

\begin{abstract}
A model is described in which the hippocampal system functions as resource manager for the neocortex. This model is developed from an architectural concept for the brain as a whole within which the receptive fields of neocortical columns can gradually increase but with some limited exceptions tend not to decrease. The definition process for receptive fields is constrained so that they overlap as little as possible, and change as little as possible, but at least a minimum number of columns detect their fields within every sensory input state. Below this minimum, the receptive fields of some columns are increased slightly until the minimum level is reached. The columns in which this increase occurs are selected by a competitive process in the hippocampal system that identifies those in which only a relatively small increase is required, and sends signals to those columns that trigger the increase. These increases in receptive fields are the information record that forms the declarative memory of the input state. Episodic memory activates a set of columns in which receptive fields increased simultaneously at some point in the past, and the hippocampal system is therefore the appropriate source for information guiding access to such memories. Semantic memory associates columns that are often active (with or without increases in receptive fields) simultaneously. Initially, the hippocampus can guide access to such memories on the basis of initial information recording, but to avoid corruption of the information needed for ongoing resource management, access control shifts to other parts of the neocortex. The roles of the mammillary bodies, amygdala and anterior thalamic nucleus can be understood as modulating information recording in accordance with various behavioral priorities. During sleep, provisional physical connectivity is created that supports receptive field increases in the subsequent wake period, but previously created memories are not affected. This model matches a wide range of neuropsychological observation better than alternative hippocampal models. The information mechanisms required by the model are consistent with known brain anatomy and neuron physiology.
\end{abstract}




\section{Introduction}

Since the observations of the combination of memory deficits observed in patients after surgical removal of parts of their hippocampal system [Scoville and Milner, 1957], there has been strong interest in the role of this structure in memory.

However, these and subsequent observations demonstrated three dissociations which have presented challenges to understanding the actual role of the hippocampal system. One is that although there can be severe anterograde amnesia for both semantic and episodic memory, retrograde amnesia is stronger for episodic memory. The second is that speech capabilities, general intelligence, and previously acquired skills are unaffected, despite the memory deficits. The third is that although the ability to create new declarative (i.e. semantic and episodic) memories is strongly affected, a significant ability to learn sensorimotor skills and to perform repetition priming is retained.

Furthermore, lesions to diencephalic structures such as the mammillary bodies and the anterior thalamic nuclei can generate similar combinations of deficits in the absence of damage to the hippocampal system. Thus damage to the mammillary bodies of the hypothalamus can result in anterograde memory deficits [Tanaka et al 1997], damage to the anterior thalamic nuclei can result in both anterograde and retrograde amnesia [Caulo et al 2005], but again in such cases all other cognitive capabilities are unaffected. It has also been observed that the amygdala plays a role in enhancing the memory of emotional events [Phelps 2006]. However, there is developing evidence that these structures have these effects through their action on the hippocampal system [Caulo et al 2005; Dolcos et al 2004], and should therefore be regarded as an integral part of that system.

A wide range of functional roles has been proposed for the hippocampal system to account for the observed combination of deficits. Many of these models propose two component systems to account for the combination of global anterograde semantic and episodic amnesia with stronger retrograde episodic amnesia. Typically these models have a component supporting stimulus memory and a component supporting episodic retrieval [e.g. Gluck et al, 2003], and argue that detailed stimulus information is initially registered in the hippocampal system and gradually transferred to long-term storage in the neocortex. The models in general have issues in providing an account for the full range of experimental observations [Cohen et al 1999], and do not provide any functional reason for the roles of the mammillary nuclei, anterior thalamic nuclei and amygdala other than speculation about possible redundancy [e.g. Graff-Radford 1990].

An alternative concept [Coward 1990; 2000; 2005a] is that the primary role of the hippocampal system is management of the information recording resources of the cortex. A major part of this role is determining at each point in time where information about current sensory inputs will be recorded in the neocortex, performing this function by managing a competition between all cortex areas to determine 
the most appropriate combination of locations. A side effect of this function is that the hippocampal system acquires information about which cortex locations record information at the same time, information critical for episodic memory retrieval and navigation. Retrieval of semantic memory, on the basis of associations between cortex locations that are frequently active at the same time (not necessarily with information recording), can become independent of the hippocampal system. The role of sleep includes configuration of some neocortex resources to be as appropriate as possible for recording information in the immediate future, using past experience (with a bias in favor of the most recent) as the best available estimate for future experience.

As described in this paper, the resource management concept can be developed into a detailed model that provides an intuitively simple reason for the existence of the hippocampal system, eliminates the need for complex information transfers back and forth between neocortex and hippocampus, and provides straightforward reasons for the existence of the various dissociations. An integrated account can be provided for the roles of different parts of the hippocampal system, the anterior thalamic nuclei, the mammillary bodies and the amygdala in memory. High level functional processes can be mapped into known or plausible neuron processes, for example functional learning into long term potentiation (LTP). Finally, it includes a memory related role for sleep including dream sleep that is more consistent with experiment that the alternative memory consolidation models.

Furthermore, there are system architectural arguments [Coward 2001] indicating that any system that must learn a complex combination of behaviours will tend to be constrained into an architectural form with separations between some specific subsystems. The constraints include a separation between one subsystem that records stimulus information, another that records response information, and a third that manages the resources of the stimulus recording subsystem, determining where new information will be recorded in response to a novel stimulus. The constraints require a new stimulus to be learned instantaneously (and relatively permanently) by slight expansions to the receptive fields of a small set of modules within the subsystem, but responses to those stimuli are learned gradually by continuous variation of behavioural weights assigned to the outputs from stimuli detection modules. With the neocortex corresponding with the stimulus recording subsystem, cortical columns corresponding with stimuli detection modules, the thalamus and basal ganglia corresponding with the response recording subsystem, and the hippocampal system with the resource manager, the dissociations in damage following hippocampal system damage can be understood, because although no new stimuli can be learned, the behavioral associations of existing stimuli are preserved and can continue to change. Knowledge acquired by the resource manager about which columns recorded information at the same time can be used to reconstruct episodic memories, but not memories based on frequent simultaneous presence of different stimuli (i.e. semantic memories such as word meanings).

This paper presents a detailed model of hippocampal functions based on the resource management concept. Firstly, a range of previous models for the role of the 
hippocampus are described. Then the architectural constraints on complex learning systems (as described fully in Coward [2001]) and how they apply to the brain are outlined. The differences between the information models for semantic, episodic, priming and procedural memory that follow from the architectural constraints are described. Next, an overview of the relevant physiology of the hippocampal system is presented. The proposed resource management model is then described, including the roles of each structure in the hippocampal system, the roles of the participating hypothalamic, thalamic and amygdala nuclei, and the role of sleep including REM sleep. The way in which memory processing occurs as a sequence of detailed physiological steps is then described. The resource management model is used to provide an account for a range of experimental observations in human beings and various animal models, and comparisons are made with various alternative models.

\section{Models of Hippocampal System Function}

In 1957, Scoville and Milner reported on a striking combination of memory deficits that had followed surgical resection of the medial temporal lobes, including substantial hippocampal damage, in three patients DC, HM and MB. In each case the patients appeared to have lost the ability to remember any events subsequent to their surgery, and memory of events for a period of time prior to surgery was also affected. However, general intelligence, conversation skills, perception and reasoning ability appeared unaffected. The details of the deficit in patient HM have been extensively investigated in the half century since than [Corkin 2002].

The deficit in HM included global anterograde amnesia for declarative type memories: an inability to learn any new events, facts or words. There also appeared to be a retrograde deficit for memory of events. In the 1957 paper it was remarked that in conversation with HM a couple of years after his operation, he reverted constantly to boyhood events and appeared to have a partial retrograde deficit "inasmuch as he did not remember the death of a favorite uncle three years previously...yet could recall some trivial events that had occurred just before his admission to the hospital". Later, HM was tested more formally using Crovitz's test, in which subjects are asked to relate a personally experienced event incorporating each of ten nouns. HM's memories were only of events earlier than 11 years prior to his operation, in striking contrast with normal controls [Sagar, Cohen, Corkin and Growdon 1985]. This apparent 11 year retrograde deficit in episodic memory contrasts with his retained semantic memory for word meanings learned in the same 11 year period [Kensinger, Ullman, and Corkin, 2001].

However, HM retained a significant ability to learn sensorimotor skills [Corkin, 1968] and his repetition priming capability was normal [Milner, Corkin and Teuber, 1968]. 
Given the association of the hippocampus with navigation [e.g. Maguire et al 2000], it is of interest that immediately after surgery, HM "could no longer ... find his way to the bathroom" and after moving to a new house a few blocks away on the same street he could not be trusted to find his way home alone [Scoville and Milner 1957]. His ability to recall spatial location was severely impaired [Smith 1988]. However, after living in a new home (an 860 square foot bungalow) for 8 years he was able to draw an accurate map of the location of the rooms and he retained that capability three years after leaving that home [Corkin 2002].

The combination of (1) global anterograde amnesia for semantic and episodic memory, (2) the continued ability to learn simple motor skills and repetition priming, (3) retrograde amnesia for episodic memory covering limited time periods, but not for semantic memory or complex skills learned prior to onset of amnesia and (4) unaffected general intelligence etc. appears fairly typical of damage to the hippocampal region. There is, however, considerable variation in detail that will be discussed in later sections. This combination of deficits has presented a major problem for modelling the function of this region. A wide range of models has been proposed, but encounter difficulties in accounting for the exact combination of deficits that are exhibited.

Tulving et al [1996] proposed that the role of the hippocampal system is determination of the novelty of a stimulus, and encoding of current incoming information by frontal lobe cortical areas depends on the novelty of that information. This model does not address the apparent dissociation between semantic and episodic information in retrograde amnesia following hippocampal system damage. As pointed out in a review of models by Cohen et al [1999], issues with the model include observations in some cases of greater hippocampal activity for old vs. new items; other observations of differences in hippocampal activation when there was no difference in novelty; and yet other observations in which systematic variation in the degree of novelty produces no change in hippocampal activation.

The simple consolidation model [e.g. Squire and Alvarez, 1995] was developed to explain the combination of global anterograde amnesia with retrograde amnesia covering a limited period of time. In this model, information is initially registered in the hippocampal system, and gradually transferred to long term storage in the neocortex. McClelland, McNaughton and O'Reilly [1995] argued that one value of gradual transfer is that it could reduce the interference between prior and later learning.

This simple consolidation model was criticized by Nadel and Moscovitch [1997] for a number of reasons, including (1) that it does not account for the differences in retrograde amnesia between episodic memories (generally the most severe amnesia), personal semantic memories and semantic memories of public events and persons (less severe) and general semantic memory (least affected), and (2) that the retrograde amnesia period observed in some cases for autobiographic memories implies that consolidation require the entire lifetime.

Teyler and DiScenna [1986] proposed that the role of the hippocampus is to form an index of neocortical areas activated by each experienced event. Only the 
location and temporal sequencing of activated cortical modules is encoded, there is no coding of any neuronal transformation of the event itself in the hippocampus. Reactivation of the indexed neocortical modules in the appropriate spatiotemporal sequence simulates the original experience. If a new event activates only a fraction of the index of some past event, and the fraction exceeds a threshold, then the remainder of the index for the past event is activated, and this activates all the neocortical modules active during the event. Teyler and DiScenna suggested that the operation of the index could be regarded as a pattern matching function: the hippocampus continually and automatically tests each pattern of cortical activation to see if it matches previously stored patterns.

Nadel and Moscovitch [1997] extended the memory indexing theory to provide an account for the dissociations between episodic and semantic memory. In their multiple trace theory, as in the memory indexing theory, the hippocampal complex rapidly encodes all information that is attended or consciously apprehended. The hippocampal record acts as a pointer to neocortical neurons that represent the information, and binds them into a coherent memory trace. The memory trace for an episode is thus the entire hippocampal-neocortical ensemble. The difference in the multiple trace theory is that reactivation of a trace results in creation of new traces, and for semantic information some traces can become independent of the hippocampus. Damage to the hippocampal system will affect a memory to a degree dependent on the proportion of the traces for that memory that are damaged. Multiple traces within the hippocampus and traces outside the hippocampus will reduce the effect of the damage. Another approach is to argue that incremental learning and storage and retrieval of episodic memories are performed by separate subsystems of the hippocampal system. Gluck et al [2003] have claimed a synthesis of this type, with incremental learning supported by representational transformations in the input regions to the hippocampus (especially the entorhinal cortex), and the storage and recall of previously processed representations supported by the CA3 and CA1 regions.

Another two component model is that of Eichenbaum et al [1994]. These authors argue that the hippocampal system performs two sequential functions corresponding with anatomically separate structures. First, the hippocampal system can fully represent current sensory items (without relationships between them) in a memory buffer that can hold information for at least several minutes. Second, while these representations are held in the buffer, the hippocampal system compares and relates them to other memory representations, creating relational representations between the items and linking with any previously created relations involving the items. They propose that the temporary storage of sensory representations occurs in the entorhinal, perirhinal and parahippocampal cortices, and the relational processing in the CA fields, dentate gyrus and subiculum.

Lisman [1999] emphasized that a key characteristic of episodic memory is that sequences are recollected, and introduced a model in which memory sequences are recalled by a combined dentate-CA3 circuit. In this model, CA3 is a recurrent network that contains heteroassociative information making it possible for one 
item in a memory sequence to recall the next item. Long chains of such recalls are liable to become increasingly noisy and error prone. To correct such a buildup of errors, the dentate gyrus is a second recurrent network that contains autoassociative information making it possible for one item in a memory sequence to recall itself with lower noise. In this model, the role of CA1 is to convert the CA 3 output to a cortical representation, and to compare CA3 predictions of the next items in a sequence with actual sensory input derived more directly from the neocortex. As a rat moves through the place field of a hippocampal place cell, the cell fires with progressively earlier phase in successive cycles of theta activity [Skaggs et al 1996]. Lisman [1999] suggested that this phase advance is the means by which memories of successive points in time are associated. This mechanism allows association of events separated by less that about 100 milliseconds, and a buffering function (supported by neuron activity for many seconds after the stimulus is removed) allows association of events with wider separations. Lisman's model is an ambitious attempt to link processes at molecular, cellular, network and behavioural levels, but as Lisman points out, does not address how the information stored in the hippocampus is utilized by other brain networks.

\section{Hippocampal subregional information models}

As discussed in the previous section, Lisman [1999] argued that the dentate gyrus operates as an autoassociator, $\mathrm{CA} 3$ as a heteroassociator, and CA1 as a comparator. These information functions together operate to retrieve episodic memory sequences.

Kesner et al [2004] extended the pattern matching paradigm introduced by Teyler and DiScenna [1986] to attempt to understand the roles of different hippocampal regions within the framework of consolidation type theories. They identified different types of information processing, including pattern separation, pattern association, pattern completion, novelty detection, and memory (short, intermediate and long term). Pattern separation is the mechanism for separating partially overlapping patterns of activation so that one pattern can be retrieved separately from other patterns. Pattern association links patterns that are discontiguous in space or time [Wallenstein et al, 1998]. Pattern completion retrieves previously stored patterns on the basis of partial inputs.

Kesner et al [2004] review a range of experimental evidence to argue that different subregions within the hippocampal formation support different information processes. In particular, the dentate gyrus acts as a competitive network to reduce the redundancy of sensory inputs and produce sparse, orthogonal outputs. These outputs are used by CA3 to perform spatial pattern separation. CA3 supports spatial pattern association, spatial pattern completion, short term memory and novelty detection. Outputs from CA3 are used to support temporal pattern separation in CA1. CA1 performs temporal pattern association, temporal pattern completion and intermediate term memory. 
However, the way in which these information processes combine to support the memory phenomena as described by the higher level models has not been made fully clear.

\section{Architectural Model of the Brain}

As pointed out by Lisman [1999], a full theory of the hippocampus must link processes at molecular, cellular, network and behavioural levels. A critical element in such a theory is some concept of what information processes the hippocampus contributes to the rest of the brain and to the neocortex in particular. Such a concept requires a system architecture of the brain as a whole.

A general system architectural model of the brain called the recommendation architecture has been proposed by Coward $[1990 ; 2001 ; 2005 a]$, including theoretical arguments that any system which must learn a complex combination of behaviors with limited information handling resources will tend to be constrained into the forms of this model by a number of practical considerations. These considerations include the need to limit resources, the need to learn without interference with past learning, the need to recover from damage and failure, the need to construct the system itself without errors, the need for synchronicity or maintenance of associations between the results of processing the same input state (i.e. information derived from the environment or other sources at the same time) by different parts of the system, and the need to use the same resources to simultaneously process input states from different times. These practical considerations generate interacting and conflicting pressures on system architecture, and some remarkably specific architectural requirements result from the need to find an adequate compromise that satisfies these conflicting pressures.

The general architectural form of the model is illustrated in figure 1. For a complex learning system, the greater the ratio of behaviors to resources, the more tightly the system will be confined within this architectural form [Coward 2001]. As illustrated in figure 1, there are a number of separations between subsystems which perform different types of information processes, and evidence from physiological structure, dissociations between different cognitive processes, and the deficits resulting from local damage has been offered [Coward 1990; 2000; 2005a] to support the view that there is a correspondence between these subsystems and the physiological structures of the mammal brain identified in the figure.

The model makes it possible to create a hierarchy of causal descriptions of the same phenomenon at a number of different levels of detail, from physiological to psychological, in such a way that descriptions on one level can be mapped into descriptions on other levels. Such a description hierarchy is essential for understanding a complex phenomenon [Coward and Sun 2007].

In the architectural form illustrated in figure 1, there is a primary separation between a modular hierarchy (called clustering) and a component hierarchy (called 
competition). The difference between a module and a component is that component inputs and outputs can only have simple behavioral meanings (i.e. recommending performance of one behavior, or against performance of anything except one behavior). Module inputs and outputs can have complex behavioral meanings (i.e. recommending performance of many different behaviors, generally with different recommendation strengths) [Coward 2001; 2005a]. In the mammal brain the cortex corresponds with the clustering and the thalamus and basal ganglia correspond with competition.

Clustering defines and detects information conditions within the information available to the system, each module being programmed to detect a set of fairly similar conditions which define the receptive field of the module. An output from a module indicates the detection of a significant subset of its programmed conditions, i.e. the detection of its receptive field. The term "feature" could perhaps be used instead of "information condition" or "module receptive field", but the implication of the word feature is that the information condition detected and resultant module output corresponds exactly with some simple cognitive feature. As discussed below, and in detail in Coward [1990; 2001], an information condition or module receptive field is a circumstance that is in general detected within many different cognitive features, the indicator of the difference between two cognitive features is the different (but partially overlapping) populations of conditions or receptive fields detected.

The system information within which conditions are detected includes raw information about the state of the external environment and about the internal state of the system itself. Competition receives inputs indicating detections of various receptive fields (i.e. module outputs) and interprets each such input as a recommendation in favour of many different behaviours, each with an individual weight. The total recommendation weights of each behaviour is determined, and competition drives the implementation of the behaviour with the largest current weight. Reward feedback modifies recommendation weights in competition but does not change condition definitions in clustering.

\section{The modular hierarchy}

A condition is ultimately defined by a set of raw system inputs and an associated state specified for each input in the set, and the condition occurs if a high proportion of its set of inputs is in the state specified for the condition. Conditions are defined on different levels of complexity, where the complexity of a condition is the number of raw inputs (including duplicates) that contribute to the condition, either directly or via intermediate conditions. Conditions on different levels of complexity (and the receptive fields which contain the conditions) may be more appropriate for recommending different types of behavior [Coward 2005a]. 


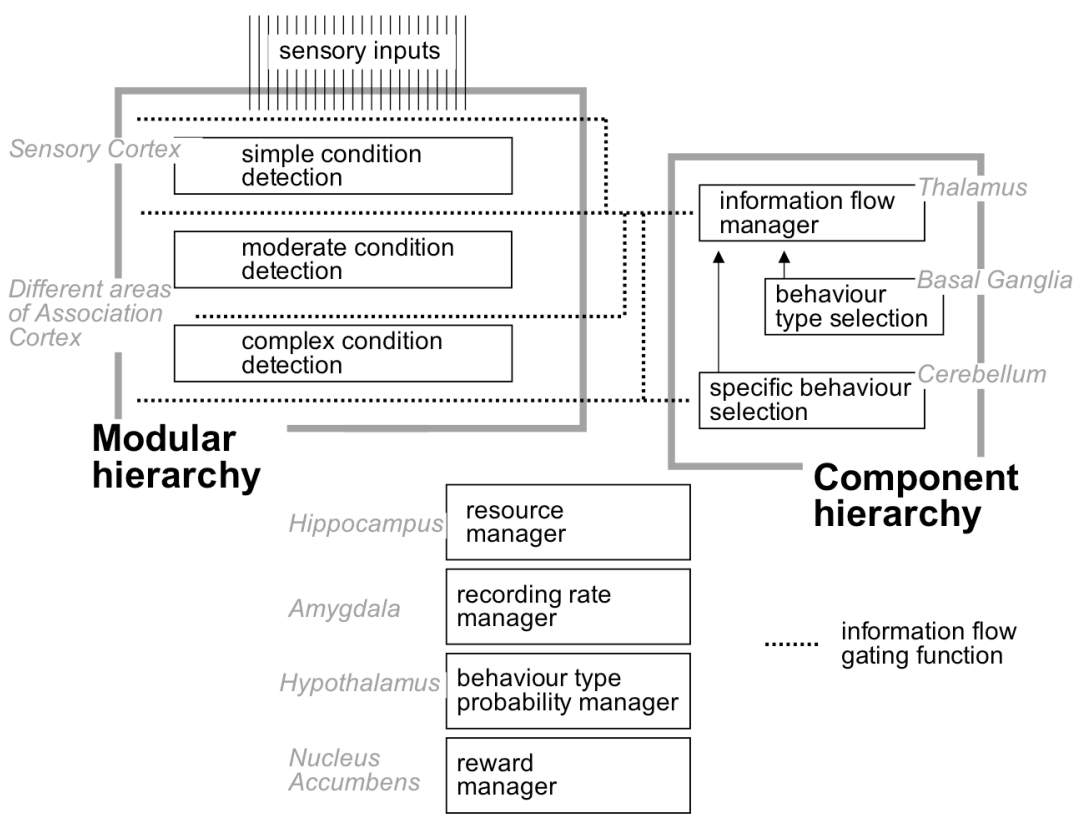

Figure 1. The architectural form into which a system will tend to be constrained if it must learn a large number of different behaviors with limited resources is illustrated. The modular hierarchy defines and detects conditions within the information available to the system. Many condition detections flow to the component hierarchy where they are interpreted as recommendations in favor of a wide range of behaviors. Conditions are detected on different levels of complexity, with simpler conditions being generally (but not exclusively) more useful for recommending information flow behaviors, moderate complexity conditions for recommending selections of general types of behavior, and high complexity conditions for recommending specific (e.g. motor) behaviors. The component hierarchy selects and implements the most strongly recommended behaviors, including behaviors that are information releases from one part of the modular hierarchy to another. Selection of, for example, a general type of behavior is effectively a selection of one information flow within the modular hierarchy over another. For efficiency reasons, most modular hierarchy information flow decisions are implemented through a single subsystem within the component hierarchy (the information flow manager), relevant selections by other parts of the component hierarchy are funneled through the information flow manager as illustrated. Reward feedback is managed by a separate subsystem. Such reward feedback acts upon the component hierarchy to change recommendation weights but cannot change condition definitions in the modular hierarchy. Decisions on where to record conditions in the modular hierarchy at each point in time are made by the resource manager on the basis of inputs from the modular hierarchy. Selection of a general type of behavior can be influenced by general circumstances via the behavior type probability manager. Such selections include influencing the rate of condition recording. Special circumstances can also result in elevation of the rate of condition recording by the recording rate manager. The human brain structures corresponding with these subsystems are indicated in red [Coward 2005a and this paper].

In physiological terms, a condition is a group of inputs to a pyramidal neuron that are integrated as a group before contributing to the potential injected into the 
soma. Figure 2 illustrates this staged integration process. A condition programmed on an arm of the dendrite is present if enough action potentials arrive at the different synapses defining the condition within a short enough period of time to exceed the threshold for injection of potential deeper into the dendrite. One neuron will be programmed with many such conditions, and will produce an output if a significant proportion of its conditions are present. The receptive field of the neuron is specified by the group of conditions that it detects, and its output indicates the detection of that receptive field.

Pyramidal neurons in sensory areas in the neocortex detect relatively simple receptive fields, with complexity increasing in later sensory areas. Pyramidal neurons in association areas detect even more complex conditions that are combinations of receptive fields detected in the areas from which they derive their inputs. Cortical columns detect receptive fields defined by the receptive fields of their constituent pyramidal neurons. Cortical arrays detect at least a minimum number of columnar receptive fields on one level of complexity in every sensory input state. Cortical areas are made up of sequences of one or more arrays, with each array detecting receptive fields on a different level of complexity but within the same input space.

An important aspect of the evolution of receptive fields is that because one receptive field has many different behavioural meanings, changes to receptive fields must be tightly controlled to minimize interference with existing such meanings. Coward $[2001 ; 2005$ a] has argued that a good first approximation is that receptive fields can expand (by addition of conditions) but cannot change or eliminate conditions once they have been added.

Two important qualifiers to this approximation are that changes to receptive fields can be reversed on very short and very long time frames with limited behavioural risk. If a condition is added but does not occur again within a relatively short period of time, it could be eliminated. In other words, the receptive field of a pyramidal neuron could expand slightly, and if the expansion is detected again soon afterwards becomes permanent, otherwise relaxing again to its prior state. If a long period of time elapses during which a condition or group of conditions do not occur, then again the behavioural risk of eliminating the condition may be low. An extreme example is if there is a major change in sensory inputs which results in some inputs no longer occurring, such as the significant changes to receptive fields observed when the surface skin of two digits of an owl monkey are connected [Clark et al 1988]. A rather different qualification is that in the early experience of the brain, condition detections may not yet be associated with behaviors. If these associations are not being created, then there can be greater freedom to change and eliminate conditions. This greater freedom may be important to heuristically establishing an effective set of modules in early learning of infants [Coward 2005a].

An implication of this restriction on change is that in general modular receptive fields cannot be evolved to correspond with features or categories of sensory objects. This is consistent with the observations of Tanaka [1993] that even in the in- 
ferotemporal cortex functionally associated with object recognition, pyramidal neuron and column receptive fields correspond with ambiguous shapes and not clear visual features.

There are a number of considerations that can be used to define the circumstances in which receptive field expansion can occur. The first is that a reasonable range of recommendations is required in response to any input state in order to achieve a high integrity behavior. Because behavioral recommendations are also module outputs, this implies that at least a minimum number of modules (i.e. cortical columns) must be producing outputs in response to every input state. If the number of columns producing outputs to the component hierarchy is low, expansion of module receptive fields by recording of additional conditions will generally be required. An implication is that the degree of condition recording will be higher during novel experiences. A second consideration is that the conditions within one module must be similar, both for resource economy reasons and to ensure that the behavioral meaning of module outputs is not excessively diluted. A third consideration is that adequate discrimination must be achieved between input states with different behavioral implications: the populations of conditions detected with such input states must be sufficiently different to adequately guide behavior. Although reward feedback cannot be used directly, there is a contradictory reward feedback mechanism that can be used to drive increases in resolution [Coward 2005a]. In this mechanism, if a similar group of columns generate outputs on a number of different occasions, these outputs result in the same behaviour being implemented, but the reward feedback following the behaviour is sometimes positive and sometimes negative, the implication is that the columns are not providing adequate discrimination. In this situation, additional discrimination could be provided (for instance, by causing two columnar output pyramidals with very similar receptive fields to diverge by forcing change to one of them). A fourth consideration is that the overall use of resources (i.e. neurons and connectivity) must not be excessive.

One approach to guiding change is to expand the receptive field of a module if the module was not producing an output, provided that the current input state was fairly similar to past input states that resulted in a module output. Another approach is to limit expansions to modules with the greatest similarity between the current input state and past state which caused them to produce outputs. This second approach means that the resultant module receptive fields are more orthogonal and therefore better able to discriminate between behaviourally different circumstances, but requires extensive communication between modules. As discussed later, both of these approaches are important.

There are some conceptual similarities between column arrays and components in independent components analysis [Hyvärinen et al 1999], in the sense that arrays decompose a sequence of input states into partially statistically independent "features" in an unsupervised manner. The critical difference is that independent components are calculated prior to use with a preselected set of sensory inputs, and do not change in response to actual sensory inputs. Columns constantly evolve 
by addition of new conditions. This evolution means that columns are less rigorously statistically independent, but new types of input states can be decomposed using an existing column array.

\section{The pyramidal neuron model}

The proposed leaky integrator model for a cortical pyramidal neuron is illustrated in figure 2 . In this model, action potentials arriving at synapses inject potential into local regions (arms) of the dendrite. These postsynaptic potentials follow a rapid increase and slower decay cycle, and add together locally within each arm. If the total arm potential at some point in time exceeds a threshold, potential is injected deeper into the dendrite. This potential also follows a rapid increase and slower decay cycle, and further integration within the dendritic tree and within the soma of the neuron determine whether the neuron will produce an output action potential. This type of staged integration across a dendritic tree appears to be physiologically plausible [Hausser and Mel 2003].

A condition is defined by inputs to one arm of the dendritic tree from pyramidal neurons with simpler receptive fields (the condition defining inputs in figure 2) and their associated synaptic weights. The receptive field of the neuron is defined by the set of conditions programmed on its arms, and the neuron will detect its receptive field if a significant proportion of its conditions are detected (i.e. inject potential deeper into the dendrite) within the integration time for the soma. The rate of action potential generation by the neuron indicates the degree of presence in the current input state of its receptive field.

Expansion of the receptive field of a neuron can take place in two ways. One is by increases to some of the input synaptic strengths of an existing condition, the other is addition of a new condition with a somewhat different set of inputs from existing conditions. It would be impractical to create the connectivity needed to define a new condition at the instant the condition was required. Configuration of provisional conditions in advance is therefore required, and condition recording occurs by increases in some of the input synaptic strengths of the new condition.

The mechanism by which synaptic strengths are increased is the LTP mechanism described by Bi and Poo [1998]. If, within the integration time for the arm, action potentials arrive at a significant proportion of the condition defining inputs, the total postsynaptic potential could exceed the threshold for the arm. If the arm injects potential deeper into the dendrite, and if shortly afterwards the soma generates an action potential, a backpropagating action potential that only enters arms which have recently injected potential into the dendrite increases the weights of recently active synapses on such arms. This mechanism will cause the receptive field of the neuron to increase whenever the neuron produces an output.

Such an unmanaged increase in receptive fields would reduce their behavioural value. There are several ways in which the increases can be managed more effectively. Firstly, by limits to the weights of individual synapses to ensure that individual synapses do not dominate a condition or individual conditions dominate a 
receptive field. Secondly, by requiring that an increase will be reversed unless several increases to the same synapse occur within a short period of time, so that rarely occurring conditions are not recorded. Thirdly, by eliminating any synapses on an arm which are close to their initial value when most other synapses have reached their maximum. Fourthly, by providing condition recording management inputs as illustrated in figure 2 , particularly to provisional conditions.

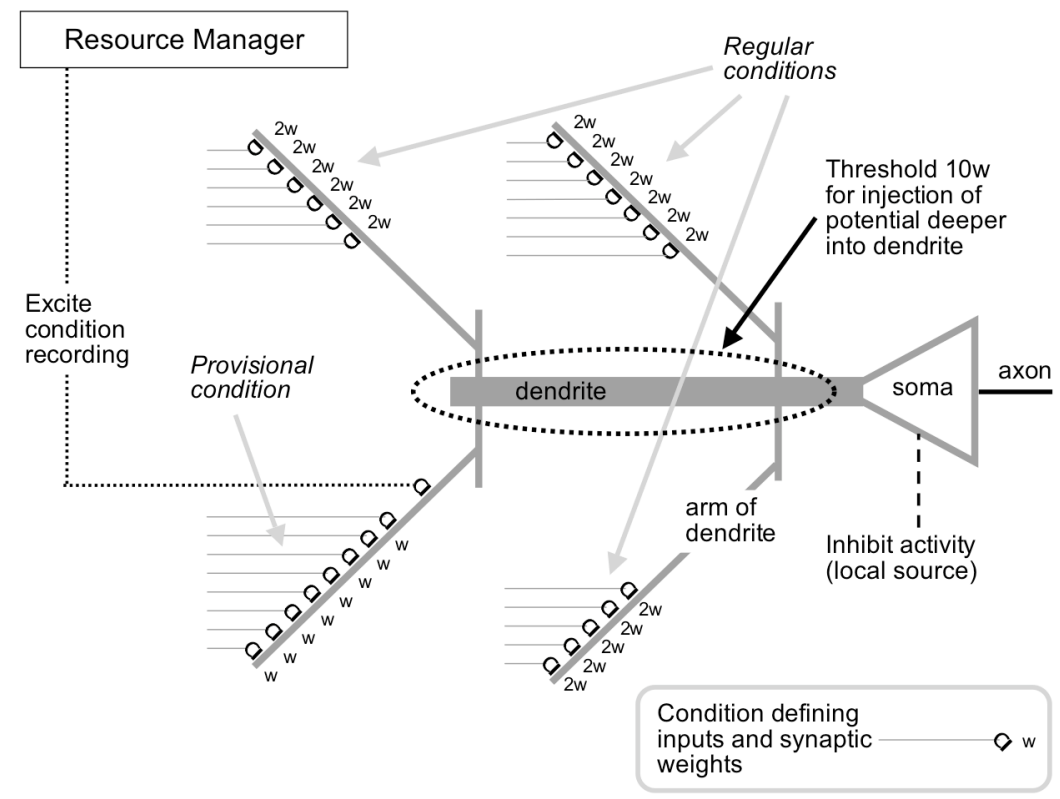

Figure 2. Conceptual diagram of a pyramidal neuron. There is separate integration of postsynaptic potentials on different arms of its dendrite. If the integration product within an arm exceeds a threshold, potential is injected further into the dendrite, and such injection from enough arms causes the soma to produce an action potential. The lower left arm does not have enough synaptic strengths in its condition defining inputs to inject potential. However, if its input exciting condition recording is active, the total potential in the arm can exceed the threshold. If shortly afterwards the neuron produces an action potential and there is also an action potential that backpropagates into arms that have recently injected potential, the postsynaptic strengths will increase by the LTP mechanism. This increase will tend to enable the arm to inject potential in the future without activity from the input exciting condition recording, in other words, a condition has effectively been recorded. Inputs exciting condition recording will in general come from the hippocampal system.

For the provisional condition illustrated in figure 2, the total weight of the condition defining synapses is in general too small to result in injection of potential deeper into the dendrite for further integration. However, if action potentials arrive at a significant proportion of the provisional condition defining inputs within the 
integration time and in addition arrive at the condition recording management inputs, the total postsynaptic potential could exceed the threshold. If the arm injects potential deeper into the dendrite, and if shortly afterwards the soma generates an action potential, the LTP mechanism increases the weights of recently active synapses on the arm. This increase means that the total synaptic strengths of those synapses would in future be enough to result in injection of potential from the arm into the dendrite independent of the state of the management inputs. In information terms, a new condition has been recorded on the neuron.

A fifth way to manage receptive field expansion uses inhibitory interneurons. If activity within a column is already at a high level, general internal inhibitory connectivity (derived from devices that received inputs from a wide range of pyramidal neurons within one layer of the column and generated inhibitory outputs) would prevent any further increase, effectively preventing recording. Such inhibitory connectivity would be directed to the pyramidal somas and perhaps to individual provisional conditions.

The critical issue of what could be an appropriate source for condition recording management inputs is discussed in the next section.

\section{Columns, arrays and management of receptive field expansions}

To understand the condition recording management process in more detail, consider the operation of the simple cortical column model illustrated in figure 3. Such columns are arranged in arrays, where the array is managed so that it detects at least a minimum number of columnar receptive fields at one level of condition complexity in all input states from a given input domain.

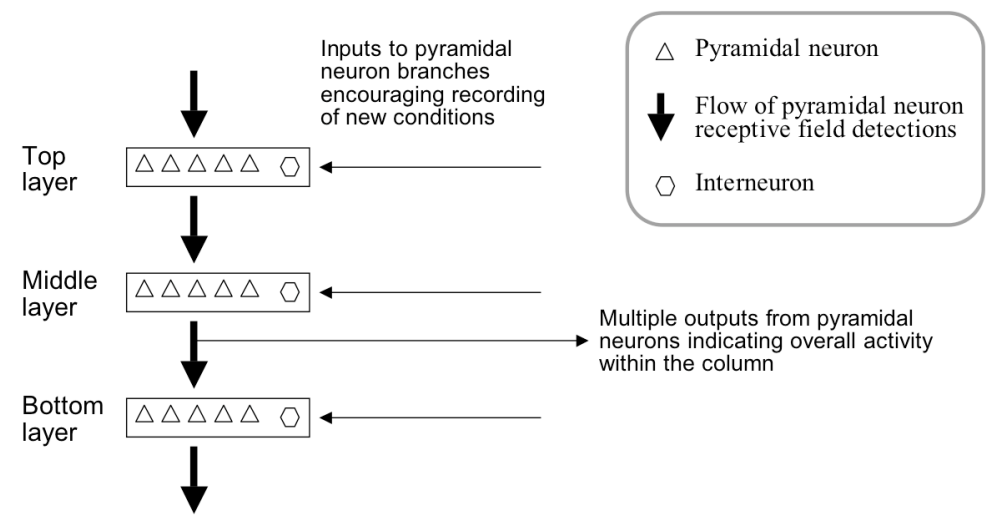

Figure 3. Column structure which could manage the condition recording process. The column records conditions in the current input state if that state is adequately similar to past input states which generated column outputs (see text). There is a competition to determine the most appropriate columns to record information in response to each input state. 
Receptive fields of pyramidal neurons in the top layer are defined as combinations of receptive fields of columns in the array providing inputs to the column. Receptive fields of pyramidal neurons in the middle layer are combinations of receptive fields detected by pyramidal neurons in the top layer, and bottom layer receptive fields are combinations of middle layer fields. There is therefore a gradual increase in receptive field complexity from top to bottom, with bottom layer pyramidals providing column outputs to the next array.

This increase in receptive field complexity means that pyramidal neurons in the top or middle layer could detect their receptive fields even if there were no such detections in the bottom output layer. However, the input state within which the middle layer detections occurred would have to have significant similarity to past input states which actually generated detections in the bottom layer. Hence if the receptive fields of some columns within an array must be expanded to reach the minimum required level of columns generating outputs, a high degree of pyramidal activity in the middle layer of one of the columns is a reasonable indicator that the needed expansion in its receptive field would be small.

To provide the condition recording management inputs discussed in the previous section, there is therefore a requirement for a process that can identify the columns with the greatest middle layer activity, and provide the pyramidals in those columns with the management signals. Such condition recording management connectivity could in principle be derived locally: excitatory connectivity from within the column, inhibitory from all peer columns. This local approach requires considerable (all-to-all) inter-column connectivity resources. Condition recording management that is equivalent in an information model sense could be performed with much less connectivity resource if every column was reciprocally connected to a global resource manager. This manager would perform a competition function to select columns to record information and generate outputs to devices in those columns to excite such recording. Local inhibitory connectivity would be required to limit internal column activity. Short range inhibitory connectivity could also implement local competition between columns with similar receptive fields to improve discrimination.

Such a global resource manager could also make use of information on which columns had expanded their receptive fields at similar times in the past to improve the selection of current columns. As discussed below, this global management function is proposed in this paper as the primary role of the hippocampal system.

At the start of sensory experience, there is a need to bootstrap receptive fields. If there were no column activity at this initial point, there would be no hippocampal activity and therefore no condition recording management inputs. Initially, therefore, receptive fields must be defined randomly (with some genetically imposed bases) and expand only on the basis of internal similarity until enough column activity is generated for the management process. Provisional conditions in the very early stages must therefore have enough condition defining input weight to lead to initial neuron activation without condition recording management inputs. 


\section{Configuration of Provisional Conditions}

Provisional conditions could be defined by random selection of their constituent conditions. However, placing a statistical bias on the selection process can increase the probable usefulness of provisional conditions. This bias is in favor of constituent conditions that have often been present in the past at the same time as each other and at a time when the target neuron has been detecting other conditions.

Such a bias is using past experience to estimate the type of conditions that are most likely to be needed in the future. The recent past is a particularly relevant guide. A simple way to achieve such a bias is to take the system off-line, and perform a rapid rerun of a sample of past activity. Provisional condition connectivity would then be created between axons indicating the presence of possible constituent conditions and target neurons being programmed with provisional conditions if input axons and target neurons were often active at the same time. Coward [1990] suggested that a primary role of REM sleep was to perform this partial rerun process. A central resource manager has a natural access to the type of information needed to guide this rerun process.

\section{Behavioral Specialization of Areas}

For maximum resource economy, a cortical array at a given level of complexity would support all possible types of behavior. However, if there were two important types of behavior for which the receptive fields at the same level of complexity that provided the best discrimination were different, the behavioral advantages of separate parallel arrays might outweigh the resource costs. In general this would occur for a limited range of receptive field complexities and for a limited range of behavioral types. Columns in one array would tend to recommend only one type of behavior, in a parallel array only a second type of behavior and so on. For example, Coward [1990] suggested that separate arrays on some levels of complexity might exist for major behavioral types such as aggressive, fearful, and food seeking. Given such separation, the arrays could be optimized for their different behavior types [Coward 2005a].

One side effect of this behavioral parallelism is that detection of general conditions indicating the appropriateness of a type of behavior could be used to bias the brain towards that behavioral type, for example low blood sugar could favor arrays generating food seeking behaviors. Such favoring could include preferential condition recording within the arrays targeted at the behavior type.

\section{The component hierarchy}

As described in Coward [2001; 2005a], the component hierarchy (thalamus and basal ganglia) is made up of components corresponding with different behaviours and types of behaviour. It receives indications of the presence of the conditions currently being detected by the modules in the modular hierarchy (cortical col- 
umns) and interprets each such indication as a recommendation in favor of a range of different behaviors, each recommendation having a specific weight. These recommendations are instantiated by the connection weights of column outputs into the appropriate components.

The component hierarchy determines the behavior with the largest total weight across all currently detected conditions using inhibitive connectivity between components and implements that behavior.

Components in the hierarchy correspond with individual behaviours or types of behaviours. The hierarchy must determine that one and only one behaviour (or perhaps a consistent set of behaviours) is selected in response to each input state, and that reward feedback is applied appropriately.

Coward [1990, 2000] suggested that there must be a competition between components corresponding with all behaviours, modulated so that there is one and only one "winner" producing an output, and offered evidence that the basal ganglia was the primary site for these processes. In this model, as illustrated in figure 4 , different components of the striatum correspond with different behaviours. Each component has a D1 and D2 section. The direct pathway from a striatum component D1 section to the GPi and SNr excite the behaviour corresponding with the component. If the resultant degree of excitation is either too high or too low (i.e. either multiple or no behaviour being selected), the substantia nigra pars compacta indirect pathway modulates the D2 section of the component to increase or decrease the general excitation in GPi and $\mathrm{SNr}$ until one and only one behaviour is selected. This selection is communicated through the thalamus back to the cortex, resulting in release of cortical outputs to drive the selected behaviour.

Reward feedback following a behavior affects the weights of recently active connections within the components corresponding with recently implemented behaviors. If the reward is positive, excitatory weights in those components are increased and inhibitory weights decreased, and vice versa if the reward is negative. The effect of a reward is therefore to modulate the probability of the same behaviors being selected in similar circumstances in the future. As illustrated in figure 5 , information indicating that a reward is appropriate could come from different sources corresponding with different types of situation: general (e.g. social) circumstances from the orbitofrontal cortex, emotional from the amygdala, and pain from the ventral tegmental area. These sources are integrated in the nucleus accumbens, which then drives changes to recommendation weights in the GPi and SNr.

In this competition system model, the thalamus gates the flow of information within the cortex, and the basal ganglia act by influencing the thalamus. Coward [2005a] has argued that the thalamus manages internal flows of information within the cortex, mainly on the basis of the recommendation strengths of cortical inputs directly to the thalamus, but modulated by basal ganglia inputs. For example, the basal ganglia could determine the general type of information flow (e.g. which cortical areas send outputs to which other areas), while the thalamus would determine the exact set of columns that provide those outputs. 
A

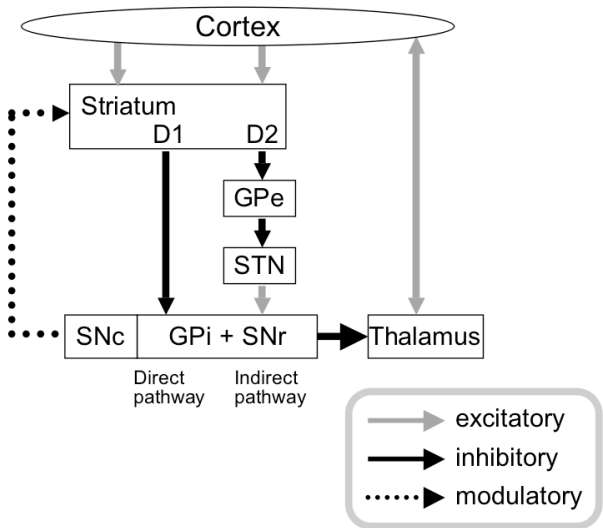

B

Same cortex inputs to both D1 and D2

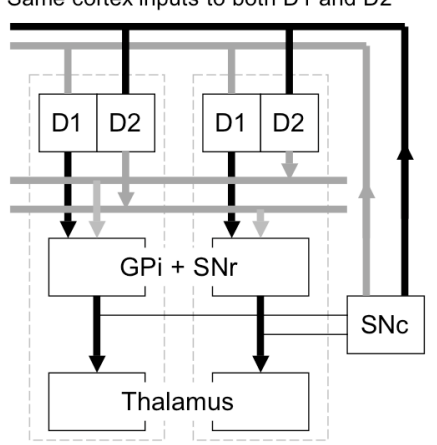

Figure 4. Basal ganglia and thalamus structures [Albin et al 1989, Alexander et al 1986] interpretated in terms of the information role of selecting behaviours most strongly recommended by the cortex. As illustrated in A, spiny neurons in the striatum receive excitatory inputs from the cortex. There are two populations of spiny neurons in the striatum. One population (D1) directly inhibits two similar structures, the globus pallidus internal segment (GPe) and the substantia nigra pars reticula $(\mathrm{SNr})$. The other population (D2) indirectly excites the same two structures via intermediate structures, the globus pallidus internal segment (GPi) and the subthalamic nucleus (STN). GPi and SNr generate tonic inhibitive outputs to the thalamus, the direct path reduces thalamic inhibition, the indirect path increases it. The thalamus receives strong excitatory input from the neocortex, and returns excitatory outputs to the same cortical areas from which the inputs were received. The thalamus provides these outputs to the cortex only if the tonic GPi and $\mathrm{SNr}$ inhibitive outputs are reduced by striatal D1 activity. A further path goes from the substantia nigra pars compacta $(\mathrm{SNc})$ back to the striatum. SNc is closely associated with GPi and SNr. The return path has different effects on the D1 and D2 populations. These structures are interpreted in terms of the information model in B. Two components corresponding with different behaviours are illustrated. The observed patch and matrix structure in the striatum [Goldman-Rakic 1982] may reflect this component structure. Each component has segments in the striatum, in GPi and $\mathrm{SNr}$, and in the thalamus. The same cortical inputs are available to both the D1 and D2 of one component. These inputs have weights that can be interpreted as recommendations in favour of the behaviour corresponding with the component. Outputs from D1 within a component can also be interpreted as recommendations in favour of the component behaviour, while outputs from D2 target other components and can be interpreted as recommendations against any behaviour other than the component behaviour. A behaviour is implemented by outputs from the thalamus that result in release of internal cortical activity either to other cortical regions or to the cerebellum, brain stem and spinal cord to drive motor behaviour. If many behaviours are strongly recommended, the SNc detects a high level of activity in GPi and SNr across all components, and increases D2 activity relative to D1 in all components until only one component has strong GPi and $\mathrm{SNr}$ activity. If GPi and $\mathrm{SNr}$ overall activity is too low, the implication is that no behaviour is currently recommended, and SNc increases D1 activity relative to D2 until activity in GPi and $\mathrm{SNr}$ supports a behaviour implementation. 
Components in the basal ganglia correspond with basic motor movements and internal cortical activation actions. However, there is also a requirement to learn and perform effectively many complex sequences of such basic movements and actions. Examples of sequences of motor movements include the sequences of motor movements required to utter words or phrases, or to perform skilled finger manipulations for typing or playing the piano. Examples of sequences of internal cortical activation actions include speech generation and generation of episodic memories.

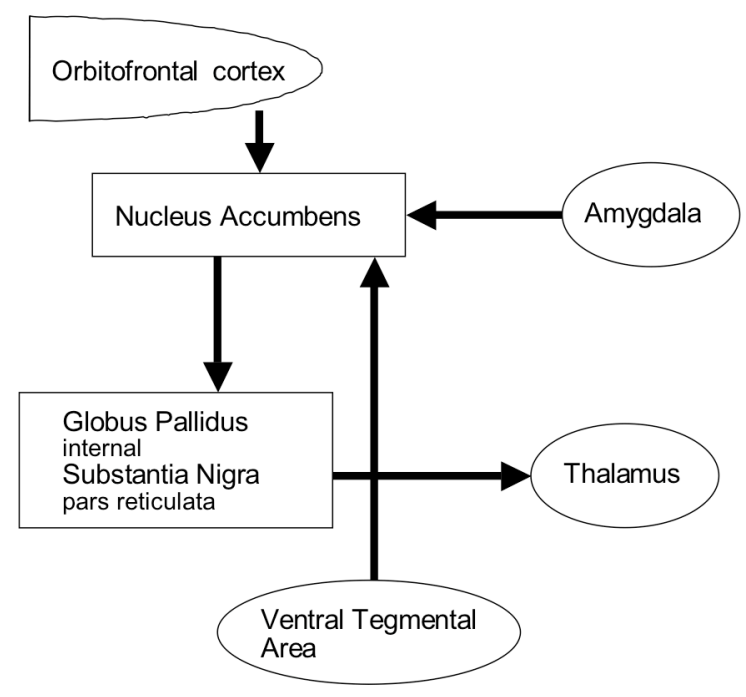

Figure 5. Interpretation of the nucleus accumbens and associated structures in terms of the information role of modulating recommendation weights of recently selected behaviours. The nucleus accumbens has been associated with positive rewards [Kelley 1999] and negative rewards [Schoenbaum 2003]. The nucleus accumbens receives strong inputs from the orbitofrontal cortex and the basolateral amygdala [McDonald, 1991; Haber et al 1995], and from the ventral tegmental area [Sesack and Carr 2002]. The nucleus accumbens projects strongly to the globus pallidus and substantia nigra [Nauta et al 1978]. In terms of the information model, the orbitofrontal cortex provides inputs correlating with general circumstances in which rewards are appropriate (e.g. detection of social signals). The amygdala provides inputs correlating with somewhat more specific circumstances, and the ventral tegmental area provides inputs correlating with very specific circumstances (e.g. perception of pain). The nucleus accumbens integrates these inputs and provides signals that modulate the connection weights of inputs to GPi and SNr that have recently resulted in the selection of a behaviour,

There is therefore a requirement for a subsystem which can learn to perform such sequences. Such a subsystem makes it possible to recommend, select and reward such behavioural sequences as a whole, making it less likely that their performance will be inappropriately interrupted, and Coward [2005a] has proposed that the cerebellum performs this function in the mammal brain. If invoked, such a 
sequence component biases each individual behavioral component (located in the basal ganglia or thalamus) in turn. The effect of biasing the first component is that the weights of currently active cortical columns in favour of the corresponding behaviour are favoured, and if there is a reasonable level of such weight the behaviour will tend to be implemented. Once the first behaviour has been performed, the component corresponding with the second behaviour is biased and so on. The behaviour sequence will therefore proceed only if there is enough total recommendation weight of the appropriate type in the active column population, but there will be a tendency to hold off from other behaviours until such a total is present. Damage to such a subsystem will not remove the ability to perform behaviours, because the individual behavioural components are driven directly by cortical outputs. However, such damage would result in problems with detailed coordination. This type of problem is a typical result of damage to the cerebellum [Gilman et al 1981].

Indirect activation behaviours will also benefit from this coordination function. One example is the sequence of activation behaviours for activating episodic memories as described in the next section. Many such indirect activation sequences will be needed to support different types of cognition, and as expected for this model, the cerebellum appears to have a role in language and cognitive functions [Liener et al 1993].

\section{Memory and indirect activation of information}

The relatively permanent recording of information in cortex columns makes a number of information activation mechanisms behaviorally useful. Suppose that a set of columns is producing outputs because they are detecting conditions within current sensory inputs. There may be other columns that are currently inactive but which may have recommendation strengths relevant to the current circumstances. For example, if a column is inactive but has recently been active at the same time as many currently active columns, or has often been active in the past at the same time as many currently active columns, or has recorded conditions in the past at the same time as many currently active columns, it may have relevant recommendation strengths.

A column may therefore have recommendation strengths in favor of activation of other columns on the basis of temporally correlated past activity. Such a capability makes it possible to expand the information available to guide behavior beyond that present within current sensory inputs. As discussed in Coward [2005a; 2005b], indirect activation on the basis of frequent past simultaneous activity makes semantic memory possible, activation on the basis of simultaneous past recording supports episodic memory, and activation on the basis of recent activity supports priming.

In the case of semantic memory, hearing a particular word (e.g. "bird") activates a fairly consistent set of auditory columns, while seeing different instances of a category of objects (e.g. different types of bird) activates different sets of vis- 
ual columns with a fair amount of overlap on some levels of condition complexity because of visual similarity. The auditory columns will therefore often be active at the same time as a frequently occurring subset of the visual columns. Hearing the word "bird" will therefore (on the basis of frequent past simultaneous activity) generate a visual activation at some levels of condition complexity as if an "average" bird were being seen. Conditions close to visual inputs will not be active, so there will not be a visual hallucination.

In the case of episodic memory, during an experience there will be some degree of condition recording in a range of columns that may be otherwise relatively unrelated. The degree of condition recording will be particularly high during an experience with a significant degree of novelty. Subsequently, activation on the basis of past temporally correlated condition recording could partially reconstruct the pattern of column activation during the experience, resulting in an episodic recollection. As an example, consider episodic memory of watching news of the first Bali Bombing (as discussed in Coward [2005a]). At the time of the original experience, there would have been considerable novelty in the sensory experience, and therefore considerable information recording in the columns active at the time. If later the words "Bali" and "bombing" were heard, they would generate activity on the basis of frequent past simultaneous activity of the auditory and visual columns. There might be some overlap with the population active during the earlier experience, but probably not enough to support verbal recall. However, if this active population were evolved on the basis of past simultaneous condition recording, it would tend to move towards an approximation to the population active at the time of the original experience, in other words an episodic recollection. Evolution on the basis of recording shortly after the current population makes it possible to move through the experience.

Viewed from this perspective, navigation is closely related to episodic memory, since it depends on learning what visual experiences come at the same time and after other visual experiences, based on prior visual experience.

A resource manager function that selected the columns to record conditions at each point in time would have a natural access to the information required to activate columns in the future on the basis of temporally correlated condition recording, but no such natural access to information required to activate on the basis of frequent simultaneous past activity. Such a resource manager can therefore be expected to play a role in creation of both episodic and semantic memories, but long term only in access to episodic memories. For example, when a category name is first learned, the link between the word and the visual information that defines its meaning would be on the basis of simultaneous auditory and visual information recording. Once the word has been used a number of times, the basis of the link will shift to frequent past simultaneous activity of that information.

In the case of procedural memory, information is recorded in the receptive fields of columns and in the recommendation weights of those columns into the thalamus and basal ganglia. Access to previously learned skills requires activation of the relevant columns and interpretation of column outputs as recommendations 
in the thalamus and basal ganglia. Because skills are learned by a process of repetition, there may be a requirement for indirect activation on the basis of frequent past simultaneous activity, but not for indirect activation on the basis of past simultaneous recording. A new simple skill in a familiar motor domain could possibly be learned by changes to column recommendation weights alone, but learning a more complex skill would also require changes to column receptive fields.

\section{Resource Management Function}

The brain model proposed on system theoretical grounds thus has a requirement for a resource management function with a number of primary roles. One role is to assign cortex condition recording resources, including additional provisional conditions to devices, additional device resources to columns and additional columns to arrays etc. A second role is to manage the configuration of the resources by ensuring that assigned resources have connectivity to and from the appropriate targets and input sources. A third role is to determine which columns will record conditions at each point in time. This third role includes biasing condition recording towards arrays generating recommendations in favor of currently selected general behavior types and to increase the degree of condition recording in circumstances determined to be critical.

In this resource management model, the hippocampal system receives inputs indicating the degree of internal activity within each cortical column. However, there is another source of information which could be used to improve the selection of the most appropriate columns. Suppose that there is some set of columns that have often expanded their receptive fields at the same time in the past. Suppose further that a large proportion of the set is indicating that receptive field expansion is appropriate, In such a situation, expansion of the receptive fields of the other columns in the set could be appropriate, even if their degree of internal activity is a little smaller. An approach making use of both current activity and averaged past activity can therefore improve recording management.

The resource management has a natural access to information needed to perform a number of secondary roles. One is the support of episodic memory, but not semantic memory. A second is the detection of the degree of novelty in a situation (indicated by the overall demand for condition recording.

\section{Anatomy of the Hippocampal System}

For the purposes of this paper, the hippocampal system will be defined to include the CA fields (CA1, CA2 and CA3), the dentate gyrus, the subicular complex (subiculum, presubiculum, and parasubiculum), and the entorhinal, perirhinal and parahippocampal cortices. Another widely used term for this hippocampal system is the medial temporal lobe. The CA fields plus the dentate gyrus will be labeled the hippocampal formation. The discussion in this section will draw 
largely on work with monkeys, but work on rats and rabbits will be cited in some cases. There appear to be strong anatomical similarities between hippocampal system structures in different mammals. The CA fields contain a single layer of pyramidal cells, in contrast with the neocortex which contains three major layers of pyramidal cells. The subiculum, located physically between the CA fields and the entorhinal cortex and the CA fields, is a transition zone in terms of numbers of layers. The primary cell types in the dentate gyrus are granule and mossy cells, both generally excitatory. Inhibitory interneurons are present in all the structures.

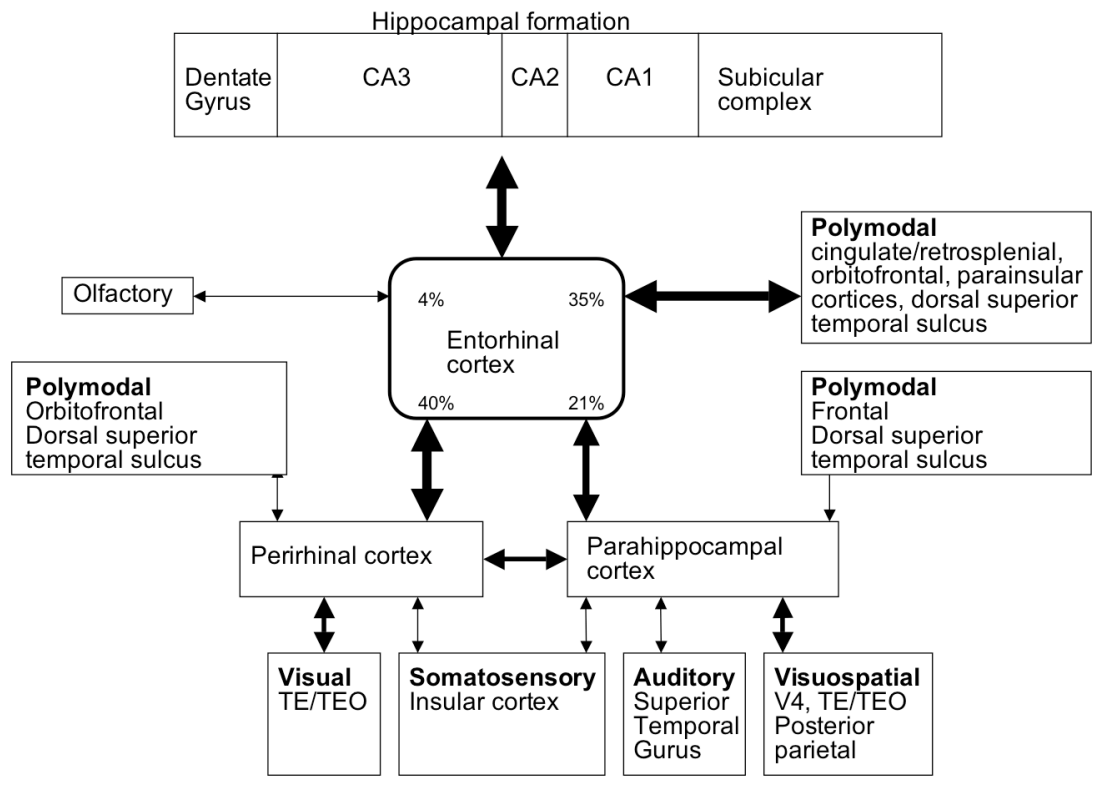

Figure 6. Neocortical sources of afferent projections to the hippocampal system. Based on Lavenex, Suzuki and Amaral 2004; Insausti and Amaral 2004; Brown and Aggleton 2001; Lavenex and Amaral 2000; Suzuki and Eichenbaum 2000; and Suzuki 1996. Percentages indicate relative volume of different sources of input to the entorhinal cortex [Suzuki 1996]. Weaker connectivity from the perirhinal and parahippocampal cortices and from some polymodal areas directly into CA1 [Suzuki and Amaral, 1990] and also into the subicular complex [e.g. Naber, Witter and Lopes da Silva 1999] is not illustrated.

As illustrated in figure 6, the extensive connectivity from the neocortex into the hippocampal system is organized hierarchically [Lavenex and Amaral 2000]. Inputs from unimodal areas are directed to the perirhinal and parahippocampal cortices. Outputs from these cortices target the entorhinal cortex, along with outputs from a number of other polymodal cortical areas. Outputs from the entorhinal cortex target the hippocampal formation. There is a high degree of reciprocity in this connectivity. For example, projections from the parahippocampal and perirhinal cortices largely reciprocate the projections from the neocortex to the parahippo- 
campal and entorhinal cortices. There is some lower degree of connectivity (not illustrated in figure 6) from the perirhinal and parahippocampal cortices and from some polymodal areas directly into CA1 [Suzuki and Amaral, 1990]. There is connectivity from CA1 directly back to those cortices. There is also some lower degree of connectivity (again, not illustrated in figure 6) from similar cortical areas directly into the subicular complex [e.g. Naber, Witter and Lopes da Silva 1999].

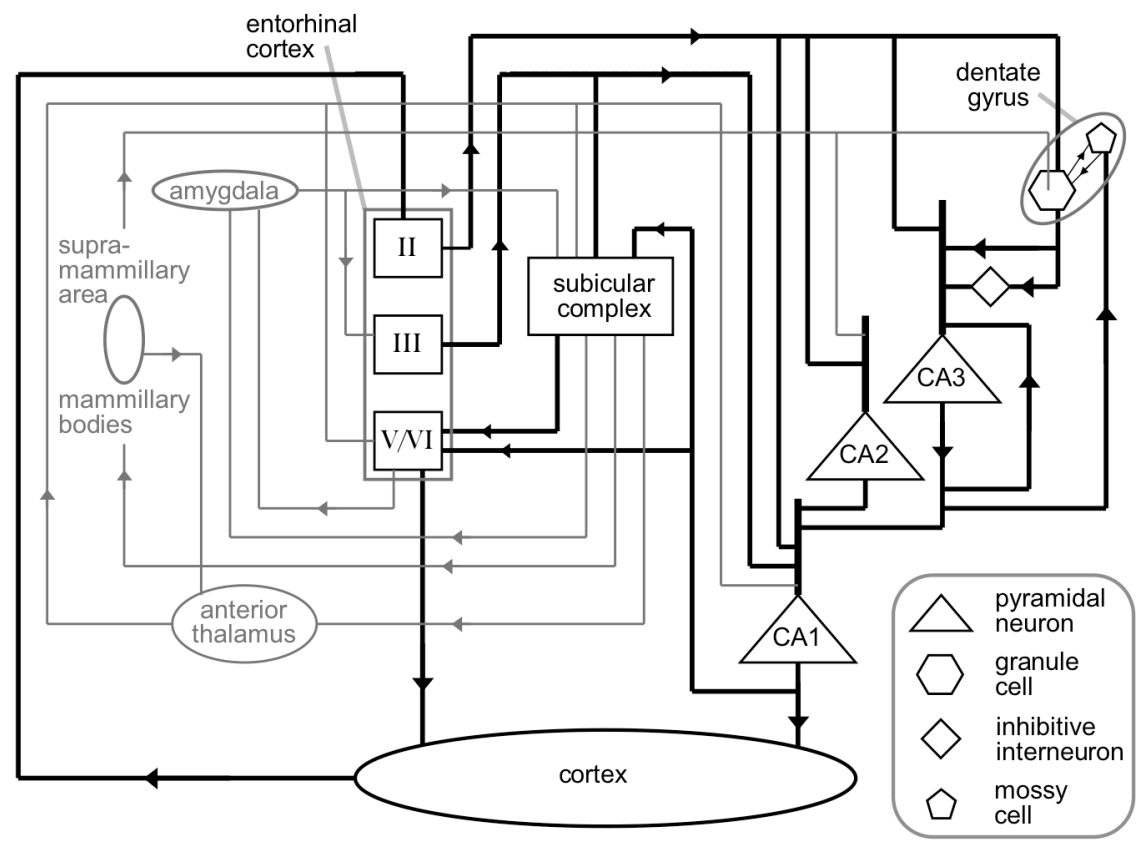

Figure 7. Major connectivity within the hippocampal formation and between the hippocampal formation and the entorhinal cortex. Also shown is the connectivity between the hippocampal formation and subcortical structures that have a role in declarative memory as demonstrated by the effects of damage to the structures in human patients: the amygdala, anterior thalamic nuclei, and mammillary bodies of the hypothalamus. Cortical information reaches all of the substructures of the hippocampal formation via the entorhinal cortex. Most return information to the neocortex proceeds via the entorhinal cortex, although there is a small proportion directly from CA1. The anterior thalamus receives outputs from the subicular complex and target the entorhinal cortex, the subicular complex and CA1. The amygdala receives inputs from the subicular complex and the entorhinal cortex and targets the same structures (different layers in the case of the entorhinal cortex). The mammillary bodies receive inputs from the subicular complex and target the anterior thalamus, and also target (via the supramammillary area) both CA2 and the dentate gyrus.

The major flows of information into and out of the hippocampal formation are illustrated in figure 7. Inputs from the wide range of cortical areas illustrated in figure 6 enter pyramidal layer II of the entorhinal cortex. Outputs from layer II 
target the dentate gyrus and the CA fields via the perforant path. There is also flow of connectivity from layer III of the entorhinal cortex to CA1. Most of the output from the hippocampal formation to the cortex goes from the subicular complex to layers $\mathrm{V}$ and VI of the entorhinal cortex, and from there to the cortical areas that provided input to the hippocampal system. The major exchange of information with the hypothalamus (mammillary bodies) and thalamus is also illustrated.

Information flows within the hippocampal formation and between that formation and the entorhinal cortex, the amygdala, the thalamus, and the mammillary bodies and surrounding supra-mammillary area of the hypothalamus are also illustrated in figure 7 . The three subcortical structures have been selected because, as discussed earlier, damage to these structures has been associated with memory deficits in human patients.

There is strong connectivity from CA 3 pyramidals to CA1 pyramidals, from CA1 to the subicular complex, and from CA1 and the subicular complex to the deep layers of the entorhinal cortex [Lavenex and Amaral 2000].

From the deep layers of the entorhinal cortex information flows back to the wide range of cortical areas that provided input. There is a much smaller flow of information directly from CA1 to external cortical areas as discussed earlier.

There is very prominent internal feedback in CA3. A typical CA3 pyramidal cell in the rat has about 3,600 excitatory inputs from the perforant path, but 12,000 excitatory inputs from other CA3 pyramidals [Amaral, Ishizuka and Claiborne, 1990]. It also has about 50 excitatory inputs directly from the dentate gyrus, but strong inhibitory inputs from interneurons that are much more heavily targeted by dentate gyrus outputs [Acsady et al 1998].

Outputs from layer II of the entorhinal cortex target the dentate gyrus and all the CA fields, and outputs from the somewhat deeper entorhinal layer III target only CA1 and the subicular complex [Insausti and Amaral 2004]. There is a pronounced flow of information from the (excitatory) granule cells of the dentate gyrus to both CA3 pyramidal neurons and inhibitory interneurons, with a typical granule cell innervating 11-15 CA3 pyramidal cells (in the rat hippocampus) and a much larger number of CA3 inhibitory interneurons [Acsady et al 1998]. There is some evidence for direct inhibitory (GABA) effects by granule cells on CA3 pyramidal neurons [Walker et al 2001]. Overall, it is possible to interpret the effect of a dentate gyrus granule cell as "communicating with a handful of CA3 pyramidal cells while silencing most others" [Mody, 2002].

CA3 pyramidal neurons extensively target CA1 pyramidals. A single CA3 pyramidal cell may contact CA1 cells throughout $75 \%$ of the length of the hippocampus [Lavenex and Amaral 2000], and CA2 pyramidals target CA1 pyramidals [Tamamaki et al 1988]. However, CA1 does not have the extensive internal feedback observed in CA3.

There is also very strong internal feedback within the dentate gyrus [Buckmaster and Schwartzkroin, 1994]. Granule cells excite mossy cells in the hilar region, and the mossy cells make excitatory connections back on to granule cells. As indicated earlier, granule cells have two types of target within CA3 [Acsady et al, 
1998]. Firstly, they make excitatory contacts on to the apical dendrites of a relatively small number of pyramidal cells. These contacts are to specific structures known as thorny excrescences. Secondly, they make excitatory contacts on to a wider range of CA3 interneurons, which in turn make inhibitory contacts on to CA3 pyramidals. Overall, the net general effect of the dentate gyrus on CA3 is inhibitory [Bragin et al, 1995]. CA3 pyramidal cells have axon branches that produce excitatory feedback to the dentate network, exciting mossy cells in the hilus, which in turn excite granule cells. [Ishizuka et al, 1990; Muller and Misgeld, 1991; Penttonen et al, 1997].

There are therefore two excitatory recurrent circuits, one in CA3 and the other in the dentate gyrus, with reciprocal connectivity between them [Lisman, 1999].

The amygdala provides inputs to the subicular complex and to layer III of the entorhinal cortex. The subicular complex and layers V/VI of the entorhinal cortex provide connectivity back to the amygdala. [Insausti and Amaral 2004].

The anterior thalamus receives inputs from the subicular complex via the fornix, and projects back to CA1 [Wyss et al 1979; Bayat et al 2005], and to the subicular complex and layer V/VI of the entorhinal cortex [Shibata 1993].

The mammillary bodies receive a strong connectivity from the subicular complex [Allen and Hopkins, 1989], and project strongly to the anterior thalamic nuclei over the mammillothalamic tract. The associated supramammillary area has a substantial projection to CA2 pyramidals and dentate gyrus granule cells [Veazey, Amaral, and Cowan, 1982].

\section{Hippocampal System Model}

The primary role of the hippocampal system in the resource management model is to select the cortical columns that will record information at each point in time. It performs this role by (i) collecting information on the degree of internal activity in each cortical column, (ii) processing this information to determine the relative activity of different (partially overlapping) groups of columns that have tended to record information at similar times in the past, (iii) performing a competition between the groups to determine the appropriate locations for recording, and (iv) generating outputs to pyramidal neurons in appropriate columns that drive the recording.

Various structures including the thalamus, hypothalamus and amygdala act upon the hippocampal system to modulate the selection of the appropriate cortical columns and the overall degree of condition recording. The amygdala increases the degree of condition recording above the base level in strongly emotional circumstances. In information terms, this reflects the probability that such circumstances may be more useful than average for guiding future behaviour, justifying extra information recording. The hypothalamus biases information recording in favour of cortical areas that tend to generate recommendations in favour of differ- 
ent general types of behaviour (aggressive, food seeking etc.). The bias is one way in which the probability of selection of a behaviour of the type is increased. The thalamus receives inputs from cortical columns that are interpreted as recommendations in favour of condition recording, with the weights depending upon reward feedback following such recording in different past experiences, and modulates the outputs from the hippocampus on this basis. All of these biases are implemented by changing the activity of pyramidal neurons generating condition recording management signals at some appropriate point within the hippocampal system.

The primary hippocampal role results in the collection of information that makes the hippocampal system useful for a number of secondary roles. One such secondary role is providing information about groups of columns that have recorded information at the same time in the past, to permit indirect activation of columns on this basis. This type of indirect activation is the mechanism for accessing episodic memories.

Another secondary role is supporting navigation. For every physical location, visual similarity means that a particular group of neocortical columns will have recorded conditions the first time the location was visited and will tend to record additional conditions whenever there is a novel experience while at the location. There will therefore tend to be cells in all parts of the hippocampus that correspond with different specific locations. These cells can contribute to solving navigation problems.

A further secondary role of the hippocampal system could be providing an indication of the novelty of an experience, on the basis of the overall demand for condition recording.

The hippocampal system also has natural access to information about which columns have recently recorded information, and therefore where additional resources could be required. Hence it plays a role in the assignment of such resources. Its information about which columns have recorded information at the same time as other columns is relevant to configuring those resources by creating appropriate provisional conditions.

As discussed earlier, a column can expand its receptive field if conditions are present within current cortical information that are similar enough to previously recorded conditions to avoid excessive dilution of the behavioral meanings of column outputs. "Similar enough" means that the column has produced outputs in the past in response to moderately similar cortical information. A significant level of internal column activity indicates such moderate similarity. Condition recording will be discouraged if there is already significant output from a column. Such discouragement is on the basis of activity within the same column, and could be implemented by local inhibitive connectivity.

To minimize dilution of behavioral meanings, condition recording should occur only in enough columns to meet the minimum required total column activity (i.e. the level at which there is an adequate range of behavioural recommendations to support a high integrity behavioural selection). These columns should generally be 
those with no outputs but high levels of internal activity. An all-to-all competition is required to determine the identity of those columns, which in connectivity terms is most efficiently performed by a central resource manager as discussed earlier. However, if this simple competition is biased in favor of groups of columns that have recorded conditions at the same time in the past, the chance of identifying a consistent group with minimum dilution of behavioral meanings is improved.

The requirement is therefore to perform a competition between different groups of cortical columns on the basis of current activity within the group and the degree to which the group has tended to record conditions at the same time in the past. Any one column could of course appear in multiple such groups. The competition determines the identity of groups of columns to record conditions at each point in time, and a translation back into condition recording management signals directed to the pyramidal neurons in individual columns that appear in many of the selected groups is then required.

Computer simulations have been performed using three layer columns, a very simple pyramidal neuron model with binary inputs and binary outputs, and a learning algorithm in which receptive fields expand but do not contract. These simulations demonstrate that a set of columns employing a competitive process based on middle layer activity can self organize to discriminate between input states with behaviourally different implications [Gedeon et al 1999; Coward 2001; Ratnayake et al 2003]. Such self organized columns can be used in combination with a simple basal ganglia model to learn high integrity behaviour selections that are resistant to interference between prior and later learning [Coward et al 2004].

\section{Physiological operations supporting memory functions}

This resource manager model can be understood in more detail by consideration of the major physiological connectivity routes as illustrated in figures 6 and 7 . In the model, as in figure 6 , information on the internal activity of cortical columns (i.e. activity of pyramidal neurons in an appropriate middle layer) is communicated to the parahippocampal and perirhinal cortices. Conditions programmed on pyramidal neurons within these cortices are inputs from sets of cortical columns that recorded conditions at the same time in the past, and the receptive fields of columns in these parahippocampal and perirhinal cortices are therefore groups of cortical columns that have often recorded information in the past at the same time. These groups will generally be limited to columns within one cortical area. Outputs from these parahippocampal and perirhinal columns target the cortical columns providing their inputs, and constitute the management inputs that excite condition recording to those columns. These outputs are generally not activated without inputs derived from the hippocampus via the entorhinal cortex.

Outputs from another layer of parahippocampal and perirhinal columns target the entorhinal cortex. In the entorhinal cortex, columnar receptive fields resulting from these parahippocampal and perirhinal inputs are groups of groups of cortical 
columns that have recorded information at the same time in the past. These groups of groups will generally include columns in multiple cortical areas. Entorhinal columnar outputs from one layer target the parahippocampal and perirhinal columns providing their inputs, and constitute the management inputs that excite condition recording in those columns. These outputs are not activated without inputs derived ultimately from the hippocampal formation. Entorhinal outputs from another layer are provided to all of the components of the hippocampal formation.

A high level of entorhinal input to the hippocampal formation thus reveals a strong activation of cortical columns in response to the current input state without any changes to receptive fields, indicating that the state is relatively familiar and a low level of condition recording is appropriate. Conversely, a low level of entorhinal input indicates a novel situation, requiring a high level of condition recording in order to activate enough columns to achieve an adequate range of behavioural recommendations. Note that for a given sensory input state there could be both familiar and novel aspects: for example individual sensory objects could be familiar but their spatial arrangement could be novel. The activity of the entorhinal cortex would carry all this information. A competition occurs within the hippocampal formation to determine the groups of columns most appropriate for recording information, and the hippocampal output structure (the subicular complex) begins the conversion of the outputs of this competitive process into signals that can drive recording. This conversion process continues back through the cortices associated with the hippocampus to the sensory, association and motor cortices.

The competition process can be understood by consideration of the physiological connectivity illustrated in figure 7 . Input from the entorhinal cortex comes into granule cells in the DG. These cells detect conditions that indicate activity of groups of groups of groups of cortical columns. Dentate gyrus granule cells do not have condition recording management inputs, and their receptive fields therefore develop without the benefit of the management process. As a result, these receptive fields will be relatively poorly focussed on groups of entorhinal cortex columns that tend to be active at similar times.

DG granule cells have two types of target in area CA3. Firstly, they directly excite specific structures (thorny excrescences) on the dendrites of those CA3 pyramidals that have similar receptive fields to the source granule cells. These structures define provisional conditions on the CA3 pyramidals that are combinations of entorhinal inputs, and the DG inputs are functionally the inputs that excite condition recording. Secondly, they excite CA3 interneurons that in turn inhibit a wider range of CA3 pyramidals that have different receptive fields from the source granule cells. CA3 pyramidal neurons also have large numbers of excitatory inputs from other CA3 pyramidals. CA3 pyramidal outputs target granule cells in the DG, and also CA1 pyramidal neurons.

If there is strong input from the entorhinal cortex, the implication is that the input situation is familiar and little information recording is required. In this situation, granule cells will be strongly excited, generating strong CA3 interneuron ac- 
tivity, which will prevent significant CA3 pyramidal activity. If entorhinal cortex input is weak, there will be relatively weak activity by granule cells, and weak activity of CA3 interneurons. Initial CA3 pyramidal activity is driven by inputs from the entorhinal cortex and indicates detection of the activity of groups of groups of groups of cortical columns. Direct input from granule cells triggers recording of additional conditions. Feedback from other CA3 pyramidals biases activity in favour of groups of groups of groups that recorded information at the same time in the past. The effect is to activate a population of CA3 pyramidals corresponding with a set of groups of groups of groups of cortical columns that have all tended to record information at the same time in the past. Condition recording on the CA3 pyramidals will slightly expand their receptive fields to include groups about to record information at the same time. As CA3 pyramidal activity increases as a result of condition recording, feedback to DG granule cells via mossy cells increases, and the resultant increased activity of the granule cells increases the inhibition back into CA3 and limits the buildup of CA3 activity. The larger the input from the entorhinal cortex, the smaller the total CA3 activity. In other words, CA3 activity will be proportional to the degree of novelty in the current input state.

CA1 pyramidals receive inputs from the entorhinal cortex and detect conditions that indicate activity of groups of groups of groups of cortical columns. The use of condition recording management inputs derived from DG granule cells means that CA3 pyramidal neurons will have receptive fields more sharply focussed than DG granule cells on groups of columns that tend to have recorded information at similar times in the past. Outputs from CA3 pyramidals target CA1 pyramidals with similar receptive fields (i.e. that have often been active in the past at the same time), and both directly excite those pyramidals and form their condition recording management inputs. CA1 pyramidals thus take the results of the CA3-DG competitive process and generate stable outputs that drive receptive field expansions throughout the cortex. Because CA1 pyramidals have condition recording management inputs derived from $\mathrm{CA} 3$ pyramidals with more focussed receptive fields that DG granule cells, the receptive fields of CA1 pyramidals will be even more sharply focussed on groups of columns that recorded information at the same time in the past than the CA3 pyramidals.

Thus the staged use of management signals means that neuron receptive fields become more and more sharply focussed on groups of columns that have tended to record information at similar times in the past, going from the very weakly focussed DG granule cells to the somewhat more sharply focussed CA3 pyramidals and then to the sharply focussed CA1 pyramidals. The CA1 pyramidals are therefore the most appropriate for driving current condition recording.

CA1 outputs will not occur without appropriate inputs from the anterior thalamus, and these inputs from the anterior thalamus will be triggered by inputs to the anterior thalamus from CA3 and perhaps other hippocampal structures indicating the completion of the competition.

The first step in this condition recording process is that CA1 outputs target the columns in the entorhinal cortex from which they derive their inputs. The entorhi- 
nal columns that occur most frequently in the inputs to the active CA1 pyramidals therefore receive strong inputs encouraging condition recording. These strong inputs trigger both condition recording and the generation of outputs from the entorhinal columns. The outputs are targetted on the perirhinal and parahippocampal columns that occur most frequently in the inputs to the active entorhinal cortex columns. A similar process results in condition recording in, and output generation from, the most heavily targetted perirhinal and parahippocampal columns. In turn, condition recording and output occurs in the cortical columns that occur most frequently in the inputs to the active perirhinal and parahippocampal columns.

This process results in columns in the parahippocampal and perirhinal cortices expanding their receptive fields to include the new groups of cortical columns about to record information at the same time. Columns in the entorhinal cortex add new groups of groups of columns. Pyramidal cells in CA3 add conditions that are groups of groups of groups of columns that record information at the same time, but these conditions also include information on other groups of groups of groups that recorded information at the same time in the past and are also currently appropriate for such recording. CA1 pyramidals add conditions that are groups of groups of groups of columns that record information at the same time.

DG granule cell outputs to encourage condition recording target CA3 pyramidals with similar receptive fields, and CA3 pyramidals target CA1 pyramidals with similar receptive fields. This targetting and receptive field similarity can be achieved to an adequate degree of approximation by biasing the creation of the condition recording connectivity in favour of connectivity between cells that are very frequently active at the same time. A group of entorhinal inputs that forms a provisional condition will be made up of inputs from neurons that have often recorded information at the same time in the past.

The entire information recording process occurs over a period during which the neocortical columns must be receiving a consistent set of sensory inputs (i.e. derived from a stable sensory input defined by the attention function). Time is required for flow of information to the hippocampus and back to the cortex, and time must be available to achieve the several repetitions of conditions required to create a long term LTP-supported condition recording. The implication is that memories cannot be created for visual experiences lasting less than about 100 milliseconds. The memory is fully defined in information terms at the end of the few hundred millisecond period. There may be chemical processes required to consolidate the memory [Tronson and Taylor, 2007], but the information content of the memory is not significantly changed or relocated.

Management of action potential timing is an important factor for the operation of the hippocampus. Coward $[2004 ; 2005 a]$ has proposed that action potentials generated by sensory inputs derived from the focus of attention are temporally bunched around peaks in a modulation signal corresponding with the gamma band in the EEG. This bunching means that conditions will be detected in sensory inputs derived from the attention focus but not in sensory inputs not derived from that focus and therefore not bunched. In order for the LTP mechanism to operate 
effectively, signals from the hippocampal system must arrive at neocortical columns in phase with the modulation signal. Lisman [2001] has proposed a model in which interactions between gamma and theta band oscillations can link memories of a sequence of events, and some version of this approach is required in the resource manager model.

Inputs from the neocortex arrive at pyramidals in layer II of the entorhinal cortex. Outputs to the hippocampal formation are derived primarily from the same layer. The primary outputs from the hippocampal formation are derived from CA1 and pass through the subicular complex to pyramidals in layer V/VI of entorhinal columns. These layer V/VI pyramidals generate the outputs to the neocortex.

The amygdala generates outputs indicating that a higher level of condition recording is justified because of "emotional" circumstances. These outputs target the subicular complex and layer III of the entorhinal cortex, and have the effect of increasing the level of output to neocortical columns selected for condition recording. This increase is limited to such columns within specific cortical areas recording complex associative conditions more likely to be useful in the future that simpler sensory conditions.

The hypothalamus acts on the hippocampus to bias the competition in favour of certain cortical areas that tend to result in behavioural recommendations of particular types. Outputs from the hypothalamus (supramammillary area) therefore influence the actual competition process by targetting the dentate gyrus, and increase the degree of recording in the target areas by targetting CA1 pyramidals. The role of the CA2 field is to receive inputs from the supramammillary area reflecting a selection of a general behavioral type to receive priority. CA2 then biases the CA1 selections in favor of cortical areas that tend to generate recommendations of the selected behavioral type. Feedback on the degree of current output from the subicular complex to the mammillary bodies regulates the degree of total output. It is also important to note that one source of information that the hypothalamus can use to select appropriate behavioural priorities is the relative degree of activity in different cortical areas. This information is therefore provided to the mammillary bodies over the fornix.

The thalamus in general gates the flow of information between different cortical areas. These information flows ultimately result in behaviours, and the thalamus uses reward feedback following behaviours to modulate the probability of similar information flows in the future. The anterior thalamus therefore excites the structures generating condition recording outputs (CA1, the subicular complex and layer V/VI of the entorhinal cortex) to different cortical areas. The type of behaviour currently favoured is relevant to the selection of information flows, and there is therefore connectivity from the mammillary bodies to the anterior thalamus.

An input state that is sufficiently novel at the sensory level will require information recording in the relevant primary sensory cortex, and recording in other areas is not an alternative (although it may well occur in addition). Hence decisions on information recording in primary sensory areas could be made locally without reference to the central resource manager, because connectivity to support such 
local decisions would not be excessive. This would account for the exclusion of the primary sensory areas from the cortical regions providing input into the hippocampal system (figure 7).

\section{Creation of connectivity}

At a number of points in the description of the resource manager, the existence of appropriate connectivity has been implicitly assumed. One key requirement is for connectivity to support provisional conditions. Recorded conditions must be similar to other conditions already recorded on the same neuron, where "similar" means that they share inputs with and/or occur at the same time as previously recorded conditions. Inputs to provisional conditions could be selected randomly from the same sources as existing conditions, but this approach risks wasting a high proportion of connectivity and including irrelevant inputs that happened to be active at the time of recording but are rarely active at the same time as the other inputs. However, information about past temporally correlated activity can be used to improve the probability of creating useful conditions [Coward 1990; 2000].

For example, suppose that the cortex and hippocampal system were submitted to an internally generated activation that was a weighted average of past condition recording activity with a bias in favour of more recent activity. Then suppose that new dendrite arms accepted inputs from groups of axons in their neighbourhood, provided that those axons tended to be active at the same time. Such provisional conditions would have a significantly higher probability of generating useful regular conditions. Simulations have demonstrated that this type of approach reduces the required connectivity in cortical models using simple binary input and output neurons by about 20\% [Coward 2000]. Coward [1990] proposed that one role of sleep is to support the creation of provisional connectivity, with REM sleep providing the weighted average rerun of past activity required to identify the most appropriate connectivity. In this proposal, unlike consolidation models [Squire and Alvarez, 1995], there are no changes to past memories. The effect of REM sleep is to create connectivity that is as appropriate as possible for recording information in the future, using a weighted average of past experience as the best available estimate for future experience. The averaged rerun will be required both in the hippocampal system and throughout the neocortex to support configuration of provisional conditions.

Similarity of receptive fields of two neurons means that the two neurons will have a high tendency to be active at the same time over an average of past experience. The rerun can therefore be used to support creation of the required appropriate connectivity between granule cells and CA3 pyramidals, between CA3 pyramidals and granule cells, and between CA 3 pyramidals and CA1 pyramidals on the basis of receptive field similarity. Furthermore, the creation of appropriate connectivity between $\mathrm{CA} 3$ interneurons and $\mathrm{CA} 3$ pyramidals can be managed on the basis of low tendency to simultaneous activity of the CA3 pyramidal and the granule cells providing inputs to the interneuron. 
Because the receptive fields of hippocampal neurons contain information on groups of columns that have recorded information at the same time in the past, the system is the appropriate place to drive the rerun activity both within the hippocampal system and throughout the neocortex

\section{Access to memories: episodic memory}

As discussed earlier, episodic memory depends upon indirect activation of neocortical columns on the basis of simultaneous past receptive field expansions. Activation on this basis generates an overall active column population that approximates to the one active during the experience that is being recalled. The hippocampal system preserves information on such past simultaneous information recording, and it therefore contains the information needed to drive access to episodic memories.

Pyramidal neurons in the parahippocampal, perirhinal and entorhinal cortices, and in CA1 and CA3 all contain information on columns that recorded information at the same time in the past, as do granule cells in the dentate gyrus. However, CA1 pyramidal cells are the primary driving force for information recording, and use of these cells for access to episodic memories risks recording of irrelevant information.

The mechanism for accessing an episodic memory is a sequence of steps. Firstly, an initial neocortical column population is activated by, for example, hearing trigger words. The columns in this population have recommendation strengths in favour of indirect activation of other columns on the basis of simultaneous past condition recording. These recommendation strengths are instantiated by connection weights of column outputs into components of the thalamus and basal ganglia that correspond with the behaviour type. Such recommendation weights are set relatively high at the moment that simultaneous recording occurs, and decay with time. Use of the recommendation strengths, particularly if followed by positive reward feedback, blocks the decay and even increases the weights.

The same columns have many other types of recommendation strengths, and the second step is a competition within the thalamus and basal ganglia between the different types of behaviour. If indirect activation on the basis of past simultaneous information recording is the selected behaviour, the third step is that outputs from this neocortical population are released by the thalamus to drive activation of pyramidal neurons in the parahippocampal, perirhinal and entorhinal cortices and CA3, and granule cells in the dentate gyrus. All of the activated neurons in these structures contain significant numbers of conditions made up inputs from different currently active neocortical columns. The activated neurons therefore correspond with groups and groups of groups of neocortical columns that recorded information at the same time as the currently active group of columns. Fourthly, activity in CA1 does not reach a level resulting in strong output to drive information recording because of lack of input from the thalamus. This lack of input is the result of reward feedback in similar past circumstances in which episodic memory has been 
encouraged. However, there could be a small degree of output, which would result in the ability to remember recalling the memory. Fifthly, hippocampal system activity drives activation of neocortical columns by feedback connectivity that targets pyramidal neurons in the columns from which the hippocampal system structures received input. This release will again be a behaviour managed by the thalamus. However, this feedback connectivity does not target provisional conditions, but the soma, basal dendrites or proximal apical dendrite of target pyramidal neurons, in the output layer of the neocortical columns. Sixthly, neocortical columns receiving substantial hippocampal system input of this type produce outputs. This secondary population of neocortical columns will tend to be made up of columns that all recorded information in the past at the same time, seeded by the original trigger words. This activation approximates to the activation during the past experience. Seventhly, a second cycle of indirect activation through the hippocampal system could increase the consistency of the population on this simultaneous recording basis, and therefore the degree to which the population corresponds with that during the original experience.

The receptive fields of pyramidal neurons in CA3 correspond with complex groups of groups of groups of neocortical columns from a wide range of cortical areas. Receptive fields in the entorhinal cortex correspond with somewhat less complex groups of groups, and columns in the perirhinal and parahippocampal cortices with even less complex groups from just one or a few cortical areas. The more complex the episodic memory to be accessed, the higher in the hippocampal hierarchy will be the regions that must participate. For example, retrieving an episodic memory of a complex event but including detailed sensory imagery would be expected to require participation of CA3.

Recall of a memory sequence requires activations on the basis of information recording slightly after past information recording in a currently active column population. Management of such activations requires dynamical processes similar to those described in Lisman [2001].

\section{Access to memories: semantic memory}

As discussed earlier, semantic memory requires indirect activation of neocortical columns on the basis of frequent past simultaneous activity, without any requirement for information recording. Thus the ability to access the word "Paris" from an activation generated in response to "What is the capital of France?" is based upon neocortical columns activated in response to hearing the question activating columns with recommendation strengths in favour of speaking the answer. These recommendation strengths are based upon frequent past simultaneous activity. When the statement "Paris is the capital of France is heard for the first time, there will be condition recording, and initially access to the response would be on the basis of simultaneous past recording. The degree of information recording will be much less in subsequent exposures to the information. Retrieval of the response on the basis of simultaneous past condition recording will be much less efficient 
that retrieval on the basis of past simultaneous activity. However, if hippocampal system pyramidal neurons were also required to store information on past simultaneous activation without condition recording, the additional information would make identification of the locations for new recording less effective.

Study of the deficits following local cortical damage indicates that the anterior temporal cortex is important for semantic memory [Rogers et al 2006], but functional imaging of semantic memory tasks results in activation of a very wide range of different cortical areas [e.g. Thompson-Schill, 2003]. The anterior temporal cortex can therefore be interpreted as the location within which information on frequent past simultaneous activity of different cortical columns is stored. On this model, columns in the anterior temporal cortex will develop receptive fields corresponding with groups of columns in other areas that are often active at the same time, with connectivity back to the same columns that can activate those columns without condition recording (i.e. targetting somas, basal dendrites or proximal apical dendrites rather than provisional conditions in the distal apical dendrites). Release of outputs from the anterior temporal cortex to other cortical areas will of course be gated by the thalamus. Control of access to frequently retrieved episodic type memories could of course be shifted to the anterior temporal cortex.

\section{Creation of imaginary events}

Consider the process for imagining an event that has not actually taken place, such as a party with Albert Einstein as a guest. Trigger words such as party, guest, Albert Einstein result in a pseudovisual activation on the basis of frequent past activity at the same time as the auditory columns directly activated by hearing the words. The effect is creation of a population of columns that would be activated if a party or if Albert Einstein were seen, although as discussed earlier the column activity close to sensory input would be weak and there would not be experience of a visual hallucination. However, although this population would be fairly strong at the object level of receptive field complexity (because parties and pictures of Albert Einstein have been seen in the past) it will also be weak at some of the more complex receptive field complexities level (because an event combining a party and Albert Einstein has not been seen in the past). A strong activation at this more complex level would correspond with a memory. CA1 then generates outputs resulting in condition recording that brings the weak neocortical activation up to the minimum level.

The process can be viewed as generation of an active cortical column population as though the imaginary scene was being perceived, using the same resources as would be used for recalling an actual memory, with slight expansions of receptive fields as required. This model predicts that the cortical resources used to imagine events are the same as those used for remembering similar events, as observed by Addis et al [2007]. It also predicts that, because of the loss of the ability to record new conditions, patients with hippocampal amnesia will not be able to imagine new experiences, as observed by Hassabis et al [2007]. The resource 
manager model predicts that area CA1 should be more active during imagination of events than during recollection of past events.

\section{Novelty detection}

The degree of novelty in a visual experience will be indicated by the magnitude of input to and output from the part of the hippocampal system that manages information flows with the higher visual cortices. Hence activity in that interface will indicate the degree of novelty in a visual experience.

\section{Evidence for Hippocampal System Model}

\section{Cognitive deficits following hippocampal system damage}

Physical damage to the resource manager function as described would result in loss of the ability to select the neocortical columns in which new information is recorded and to drive that condition recording. Because all existing columns and their associated recommendation strengths are preserved, there is minimal disruption to most other cognitive capabilities. However, because the hippocampal system acquires information identifying groups of columns that recorded conditions at the same time in the past in the course of its resource management role, and is therefore the natural source for information to guide indirect activations of cortical columns on that basis, there will therefore some loss of episodic memory through inability to access the information. Information to identify groups of columns on the basis of frequent past simultaneous activity or recent simultaneous activity must be collected to support semantic memory and priming. Use of the resource manager to collect such information would interfere with its primary role. Other cortical structures must therefore collect such information, and damage to the resource manager will not affect these types of memory. All existing neocortical columns and their associated recommendation weights into the thalamus and basal ganglia are unaffected by damage to the hippocampal system. Hence all previously learned physical and cognitive skills are unaffected. Recommendation weights of existing columns could still be changed by reward feedback. Hence if a skill could be acquired without changes to column receptive skills, learning of such a simple skill could proceed despite hippocampal damage. Even relatively complex skills might be acquired using a population of columns with fixed receptive fields, if past experience had accidentally provided even a suboptimal discrimination and enough repetition of the tasks occurred to establish appropriate recommendation weights. The model therefore provides a straightforward account for the striking combination of cognitive symptoms associated with damage to the hippocampal system. 


\section{Correspondence with observed physical connectivity}

As demonstrated by the description of the detailed model, the resource manager provides a functional account for all of the major connectivity paths observed within the hippocampal system and between the hippocampal system and other brain structures. In particular, it provides functional reasons for the memory deficits observed as a result of amygdala, hypothalamus and thalamus damage.

\section{Differential effects of damage to subregions}

Each pyramidal neuron in CA3, CA1, and the associated cortices preserves information identifying groups of cortical columns that have recorded conditions at the same time. The number of columns in the groups decreases from CA3 and CA1 to the entorhinal cortices and decreases further in the parahippocampal and perirhinal cortices. This information is needed for indirect activations in support of episodic memory. However, because outputs from CA1 drive condition recording, the use of CA1 for such a purpose could result in inappropriate condition recording.

Consistent with this understanding, in human subjects damage to CA1 alone generates anterograde amnesia but little if any retrograde amnesia, and no signs of significant cognitive impairment other than this loss of memory (e.g. patients RB [Zola-Morgan et al 1986] and GD [Rempel-Clower et al, 1996]). When damage extends to other hippocampal formation structures, retrograde amnesia becomes significant in addition to anterograde amnesia (e.g. patients LM and WH [RempelClower et al, 1996], patient HM [Corkin et al, 1997]).

In the resource management model, information derived from sensory experiences is recorded immediately in columns in the neocortex, and subsequent damage to the hippocampal system will not affect the information. The only effect of such damage will be on the capability to access such information on the basis of past temporally correlated information recording. If the basis for activation shifts over time towards temporally correlated activity, access will become more and more independent of the hippocampal system. For example, when a word is first learned, there will be information recording in auditory columns activated in response to hearing the word, and in visual columns activated in response to the object or concept of the word. The capability to understand the word depends upon the recorded information and in the short term, actual understanding of the word exist because the auditory columns indirectly activate the visual columns on the basis of simultaneous past information recording, utilizing information from the hippocampal system. After a number of occasions on which the word has been understood in this way, the auditory columns will acquire the ability to activate the visual columns on the basis of frequent past simultaneous activity, supported by connectivity paths within the neocortex and independent of the hippocampal system. To the degree to which this has occurred, access to the information would be expected to result in the most severe retrograde amnesia for episodic memories, less for personal semantic memories and semantic memories of public events and 
persons, and least for general semantic memory. This graduation is consistent with the observed amnesias [Nadel and Moscovitch, 1997].

An autobiographical memory is the record of complex, unique events. The link between the information active at the time of the event will therefore be temporally correlated information recording across a complex population of cortical columns, and there is no reason for these columns to be frequently active at the same time. At the other extreme, a new word is the record of a relatively simple link between auditory columns and visual columns and the columns are active at the same time each time the word is used. A shift to information access on the basis of frequent past simultaneous activity is likely to be rapid. The association between the names and faces of public individuals, and personal semantic facts represent an intermediate state. The observed graduation in retrograde amnesia with hippocampal system damage from most severe for autobiographic to negligible for word knowledge is as expected by the model. An exception could be if a particular memory were very frequently described. In such a case, frequent repetition could result in some ability to access the memory independent of the hippocampal system on the basis of frequent past simultaneous activity.

Regular autobiographical memory does not become independent of the hippocampal system. In the experiments of Rekkas and Todd Constable [2005], the activation of the hippocampal formation was observed during retrieval of both recent and remote autobiographical memories, and activity was greater for remote memories. In these experiments, the research design stressed depth of recall and encouraged visualization of details. Thus subjects were asked "Can you recall a specific high school teacher?" or "Can you recall the school yard of your elementary school?" but it was stressed to participants that the questions were meant to cue an actual episode, such as "The time the English teacher brought in a recording of Hamlet and made us listen" rather than a series of facts (like the name of the teacher). Follow-up questions like "Do you recall a time when you were playing in a specific area of the school yard?" This design aimed to exclude facts recall and recall of highly salient emotional events (e.g. weddings, graduations, loss of a pet) that may be more common in autobiographic self reports.

\section{Hippocampal system and navigation}

In the resource management model, CA1 pyramidal neurons correspond with groups of neocortex columns that have frequently recorded information at the same time in the past. CA3 pyramidals also correspond with such groups, but have interconnectivity with many other CA3 pyramidals corresponding with groups that have recorded information at the same time in the past, but somewhat less frequently. The entorhinal cortex brings together the inputs from such groups and provides inputs indicating the activity in the groups to the hippocampal formation. The entorhinal cortex also receives outputs from the hippocampal formation and translates them back into outputs directed to the individual columns to drive information recording. Hence a mapping between groups of cortical columns that 
have recorded information at the same time and individual pyramidal neurons can be expected in CA1, CA3, and the entorhinal cortex.

One situation in which recording of information at the same time can be expected is in navigation, where simultaneous recording across a specific population of cortical columns can be expected when in the same location. Pyramidal neuron "place cells" which are active when a rat is in a specific location have been observed in CA1 and CA3 fields [Leutgeb et al 2004] and in the entorhinal cortex [Fyhn et al 2004], but not in the more peripheral areas of the hippocampal system [Fyhn et al 2004]. This distribution of place fields is as expected by the model.

Furthermore, given that the role of CA 3 is to focus CA1 on an optimal group of columns to record information at the same time, place fields developed in the absence of CA3 would be expected to be less sharp, as observed by Brun et al [2002]. Changes to the environment would be expected to result in greater changes to CA3 than CA1 place fields, as observed by Leutgeb et al [2005].

\section{Damage to hypothalamus and amygdala}

In the model, the role of the hypothalamus is to influence current information recording in favor of current general behavioral priorities. Loss of this function would be expected to affect the ability to record information in the future, but to have no effect on access to past information on the basis of temporally correlated past recording. Consistent with this interpretation, damage strictly limited to the mammillary bodies (bilaterally) results in anterograde amnesia but minimal retrograde amnesia [Tanaka et al 1997]. The thalamus influences the level of hippocampal system outputs in general, and damage to the anterior thalamic nucleus can therefore result in both anterograde and retrograde amnesia as observed [GraffRadford et al 1990].

In the resource management model, the role of the amygdala in memory is to adjust the degree of hippocampal system driven information recording during emotional events in favor of cortical areas where the increased recording is likely to be useful in determining behavior in the future. Consistent with this role, it is found that emotional arousal biases the memory of the event in favor of the gist and reduces the memory for visual details, and that bilateral damage to the amygdala eliminates the bias [Canli et al, 2000; Adolphs et al, 2001; 2005]. Furthermore, it is the interaction between the amygdala and the hippocampal system that correlates with the enhanced emotional memory [Dolcos et al, 2004].

It is also relevant that, as expected by the model, the effect of lesions to the mammillary bodies and anterior thalamic nuclei is to depress the operation of the hippocampal system. Thus the hippocampal system activity observed during memory encoding and retrieval tasks in normal subjects was not observed during attempted performance of the same tasks in a subject with such diencephalic lesions but no damage to the hippocampal system [Caulo et al 2005]. Similarly in the case of emotional memory it is a correlation of activity between the amygdala 
and hippocampus that occurs during creation of stronger memories in emotional situations [Dolcos et al 2004].

\section{Role of Sleep}

In the resource management model the role of sleep including REM sleep in declarative memory is radically different from that proposed in the consolidation model. In the latter model, the role is to rerun recent sensory experiences to expedite the long-term registration of the memories of the experiences in the neocortex. The rerun must presumably be fairly precise to achieve this function. One view is that rerun of very recent experience could occur during slow wave sleep, and rerun of more remote experience during REM sleep [Hoffman and McNaughton 2002].

In the resource management model, sleep including REM sleep addresses the problem that it would not be practical to create the physical connections required to support the recording of new information at the instant such recording was needed. Rather, provisional connectivity is created in advance, and information is recorded as the most appropriate subsets of this provisional connectivity. Sleep is the period in which provisional connectivity between pyramidal neurons that could be used to record new information in the next wake period is created. Such provisional connectivity would be established between the appropriate levels of condition complexity, but if it was otherwise completely random, a high degree of connectivity would be required to ensure that subsets existed that corresponded with useful information. Much of this connectivity would be wasted and could result in significant behaviorally confusing noise. If the connectivity can be biased in favor of connectivity somewhat more likely to be useful, significant resource advantages would follow. Past experience provides the only available guide to probable usefulness. Any past experience could be relevant, but recent experience will on average be a somewhat better guide than remote experience. The proposed role of sleep is to establish provisional connectivity, but with a bias in favor of connectivity between neurons that have often been active in the past at similar times, especially the recent past. Activating neurons in a manner that reflected past temporal correlations could impose such a bias, and the presence of information on such correlations in the hippocampal system would make that structure appropriate to drive such an activation pattern. This activation pattern would be experienced as a partial, approximate rerun of past experience, with a bias in favor of the most recent [Coward 1990]. Simulations indicate that such a biasing process reduces the information recording resources required to achieve a given level of performance for a relatively simple behavioral problem by about 20\% [Coward 2001].

In the resource management model, as in the case of consolidation models, there would be a rerun of recent experience (perhaps in slow wave sleep) and more remote experience (in REM sleep). However, there would be no need for an exact rerun of past experience, only a reactivation that provides a reasonable ap- 
proximation to the temporal correlations between pyramidal neuron activities in the past. Elimination of REM sleep would not prevent learning, rather it would increase the resources required for learning by some degree.

Correlations between neuronal activity during waking and during the subsequent sleep period have been observed [Skaggs and McNaughton, 1996], and it can be argued that slow wave sleep reflects activity in recent waking experience and REM sleep more remote experience [Hoffman and McNaughton 2002]. However, although dreams include clearly recognizable waking elements, they do not reproduce real-life events [Fosse et al, 2003] as required by the consolidation models. This situation is, however, fully consistent with the resource management model. Consolidation models also have the problem that REM sleep deprivation appears to have relatively little effect on memory capabilities, and REM sleep can be substantially or completely suppressed (by various antidepressant drugs, or by bilateral damage to the pons) without apparent effect [Vertes and Eastman 2000]. In the resource management model, deprivation of REM sleep would be expected to increase the resources required for memory support to some degree, but would not qualitatively interfere with memory creation.

In the resource management model, the averaged rerun of past experience would be required to configure appropriate provisional conditions on pyramidal neurons in the hippocampal system and throughout the neocortex, including the prefrontal cortex as observed by Euston et al [2007].

\section{Comparisons with Other Models}

The central place occupied by the driving of neocortical receptive field expansions in the resource manager model makes the model qualitatively different from the alternative models discussed earlier, and conceptually much simpler. However, there are some similarities between certain functions of the resource management model and parts of other models. Indexing theory [Teyler and DiScenna 1986] suggests that the hippocampal system maintains an index of the neocortical areas activated by each experienced event. In the resource manager model, records of past simultaneous information recording by different groups of columns are maintained, but not as an explicit index for each event. However, using the records for different groups of columns as described earlier can access memories of past events. Indexing theory does not make a clear distinction between events in which there is strong recording and events in which there is just simultaneous activity. Hence the support of semantic memory outside the hippocampal system is not explained.

The multiple trace model [Nadel and Moscovitch 1997], like the resource manager model, proposes that additional memory traces are created in subsequent, similar experiences, but provides no reasons why memory traces should initially be within the hippocampal system and subsequently in a separate structure such as 
the anterior temporal cortex. The role of REM sleep is not explained in indexing theories.

Consolidation models [Squire and Alvarez 1995] propose that memories are initially registered in the hippocampal system, and over a period of time transferred to other neocortical structures, perhaps to avoid interference between prior and later memories [McClelland, McNaughton and O'Reilly 1995]. Such transfers of complex information constructs between different physiological structures would be physiologically costly and implausible, and are not required by the resource manager model, in which neocortical information records are immediately created in their long term storage locations.

Two component models attempt to account for the complex combination of memory deficits following hippocampal damage by arguing that the hippocampal system is actually two relatively independent subsystems. In the proposal of Gluck et al [2003], incremental learning is supported by representational transformations in the input regions to the hippocampus (especially the entorhinal cortex), and the storage and recall of previously processed representations supported by the CA3 and CA1 regions. It is not clear how this synthesis can be compatible with the observation that cell loss strictly limited to the CA1 field within the hippocampal system could result in the observed combination of severe anterograde amnesia and little if any retrograde amnesia [Zola-Morgan et al 1986]. In this specific case, even the perforant path connectivity from the entorhinal cortex through CA1 to CA3 and the dentate gyrus was intact. In the resource manager model, CA1 is the driving force for information recording, but plays a minimal role in retrieval, consistent with the Zola-Morgan et al observations.

Eichenbaum et al [1994] propose a two component model in which the hippocampal system contributes only to declarative memories, and plays two orthogonal roles in such memories: the temporary maintenance of memories by the parahippocampal region; and the processing of a particular type of relational memory representations by the hippocampus itself. In this model, sensory information generates a representation in the neocortex that is very sensitive to replacement by subsequent sensory inputs, and can only be maintained as long as the level of intervening interference is low. Activation of the parahippocampal region does not preserve the actual representation but creates a trace of the neocortical activation that is less sensitive to subsequent processing. Activation of the hippocampal formation processes comparisons between current and previous sensory input states. These comparisons require changes to connectivity within cortical areas, but the nature of these changes is not described in the model. Interactions between intermediate term storage in the parahippocampal region and relational processing in the hippocampus are fed back and forth for a significant period of time, contributing to the long-term consolidation of memories.

The Zola-Morgan et al [1986] observations of severe anterograde amnesia for all types of declarative memory including simple recognition memory following CA1 damage is also inconsistent with the Eichenbaum et al [1994] two component model in which the role of the hippocampal formation is limited to declarative 
memories with a strong relational content. The suggestion that the parahippocampal region plays a role in maintaining neocortical activations that are otherwise very sensitive to replacement by subsequent sensory inputs does not appear to be consistent with observations that short term memory of up to several minutes does not appear to be affected by hippocampal damage [e.g. Scoville and Milner 1957], but the dorsolateral prefrontal cortex plays an important role in working memory, appearing to direct attention to internal representations of sensory stimuli and motor plans that are stored in more posterior regions [Curtis and D'Esposito, 2003].

Wallenstein and Eichenbaum [1998] have suggested that the primary role of the hippocampus is supporting creation of memories associating items that are discontiguous in terms of their temporal and/or spatial positioning. In the resource manager model, the primary role is driving receptive field expansion throughout the neocortex, which also results in support for memories of discontiguous events. The observations that object recognition memory is also affected by hippocampal damage [Broadbent et al 2004] is consistent with the resource manager model but demonstrates a hippocampal role beyond memory for discontiguous events.

In addition to the functions of encoding, retrieval and consolidation proposed in various alternative models, some models emphasize subsidiary information processes. For example, Kesner and Hopkins [2006] argue that pattern separation, pattern association and pattern completion are important hippocampal functions, where pattern separation is defined as the ability to encode and separate events in time and space, pattern association as the ability to form arbitrary associations between events and items, and pattern completion as the ability to retrieve complete information on the basis of partial or incomplete inputs.

In the resource manager model, the hippocampal system selects an appropriate group of cortical columns to record information in response to a sensory input state, records the identities of different subgroups of the selected group that are (therefore) recording information at the same time, and uses these records both to better determine appropriate groups for future experiences and to activate groups that all recorded information at the same time in the past (i.e. to access episodic memories).

Different aspects of the resource manager model can be interpreted as functions like those proposed by Kesner and Hopkins [2006]. For example, because the visual environment is relatively stable at a particular point in space, there is a group of columns that will tend to be activated when the subject is at that point. Novel events occurring at that point in space (including the first visit to the point) will tend to result in recording of information at the same time in a group of columns that includes the group corresponding with the point. Hippocampal neurons have receptive fields corresponding with groups of neocortical columns that recorded information at the same time in the past, and some such neurons will tend to correspond with points in space. These neurons can be interpreted as performing pattern separation.

Because hippocampal neurons in the entorhinal cortex and hippocampal formation correspond with large, heterogeneous groups of neocortical columns, con- 
nected only because they have recorded information at the same time in the past, these neurons effectively perform pattern association across arbitrary associations (such as object location and object identity). The activity of these neurons can also be interpreted as pattern completion, because they can identify the larger group of neocortical columns that recorded information in the past at the same time as all of a smaller group.

Although different components of the resource manager model could be interpreted as performing the various suggested information processes, the model as a whole has the advantages that it provides an integrated account of the hippocampal system performing a single critical brain process (i.e. neocortical resource management) and a secondary process which can use the information already present in the resource manager without interfering with that information (i.e. episodic memory) implemented by detailed connectivity paths and physiological processes consistent with those observed in the hippocampal system.

\section{Conclusions}

The proposed model of the hippocampal system as resource manager for the neocortex has a number of advantages over alternative models. Firstly it is conceptually simpler, with one primary functional role accounting for all the subsystems and observed behavioural functions. Secondly, it provides a straightforward account for the specific combination of deficits observed in patients with hippocampal damage. Thirdly, it provides an integrated account for the memory related deficits observed with damage to mammillary bodies, anterior thalamus, and amygdala. Fourthly, the major connectivity paths observed within the hippocampal system and between the hippocampal system and other brain structures are as expected for the model. Fifthly, it indicates how the LTP mechanism supports the information processes required for memory. Sixthly, it provides a role for sleep in memory that has no issues with experiment. Seventhly, its role is consistent with theoretical constraints on the architectures of complex learning systems.

In general, a major advantage of the model is that it makes it possible to describe the same memory phenomenon on four different levels of detail (neuron physiology; cortical column activity; interactions between major anatomical structures; and psychology), with mapping between different levels. This mapping between different levels of description is critical for scientific understanding [Coward and Sun 2007].

\section{References}

Acsady, L., Kamondi, A., S1k, A., Freund, T. and Buzsaki G. (1998). GABAergic Cells Are the Major Postsynaptic Targets of Mossy Fibers in the Rat Hippocampus. Journal of Neuroscience $18,3386-3403$. 
Addis, D.A., Wong, A.T. and Schacter, D.L. (2007). Remembering the past and imagining the future: Common and distinct neural substrates during event construction and elaboration. Neuropsychologia 45, 1363-1377.

Adolphs, R., Tranel, D. and Buchanan T.W. (2005). Amygdala damage impairs emotional memory for gist but not details of complex stimuli. Nature Neuroscience 8, 512 - 518.

Adolphs, R., Denburg, N.L. and Tranel, D. (2001). The Amygdala's Role in Long-Term Declarative Memory for Gist and Detail. Behavioral Neuroscience 115, 983 - 992

Albin, R. L., Young, A. B. and Penney, J. B. (1989). The functional anatomy of basal ganglia disorders. Trends in Neuroscience 12, $366-375$.

Alexander, G. E., DeLong, M. R. and Strick, P. L. (1986). Parallel organization of functionally segregrated circuits linking basal ganglia and cortex. Annual Reviews of Neuroscience 9, 357 -81 .

Allen, G.V. and Hopkins, D.A. (1989). Mamillary body in the rat: topography and synaptology of projections from the subicular complex, prefrontal cortex, and midbrain tegmentum. Journal of Comparative Neurology 286, 311 - 336.

Amaral, D.G., Ishizuka, N. and Claiborne, B. (1990). Neurons, numbers and the hippocampal network. Progress in Brain Research 83, 1 - 11.

Bayat, M., Hasandeh, G.R., Barzroodipour, M. and Javadi, M. (2005).The effect of low protein diet on thalamic projections of hippocampus in rat. Neuroanatomy 4, 43 - 48.

Bi, G-q. and Poo, M-m. (1998). Synaptic modifications in cultured hippocampal neurons: Dependence on spike timing, synaptic strength, and postsynaptic cell type. Journal of Neuroscience 18, 10464-10472.

Bragin, A., Jando, G., Nadasdy, Z., van Landeghem, M. and Buzsaki, G. (1995). Dentate EEG spikes and associated interneuronal population bursts in the hippocampal hilar region of the rat. Journal of Neurophysiology 73, 1691-1705.

Broadbent, N.J., Squire, L.R. and Clark, R.E. (2004). Spatial memory, recognition memory, and the hippocampus. Proceedings of the National Academy of Sciences (USA) 101, 1451514520.

Brown, M.W. and Aggleton, J.P. (2001). Recognition memory: what are the roles of the perirhinal cortex and hippocampus. Nature Reviews Neuroscience 2, 51 - 61.

Brun, V.H., Otnaess, M.K., Molden, S., Steffenach, H-A., Witter, M.P., Moser, M-B. and Moser, E.I. (2002). Place Cells and Place Recognition Maintained by Direct Entorhinal-Hippocampal Circuitry. Science 296, 2243-2246.

Buckmaster, P.S. and Schwartzkroin, P.A. (1994). Hippocampal mossy cell function: a speculative view. Hippocampus 4, 393 - 402.

Canli, T., Zhao, Z., Brewer, J., Gabrieli, J.D.E. and Cahill, L. (2000). Event-Related Activation in the Human Amygdala Associates with Later Memory for Individual Emotional Experience. Journal of Neuroscience 20(RC99), 1-5.

Caulo, M., Van Hecke, J., Toma, L., Ferretti, A., Tartaro, A., Colosimo, C., Romani, G.L. and Uncini, A. (2005). Functional MRI study of diencephalic amnesia in Wernicke-Korsakoff syndrome. Brain 128, 1584-1594.

Clark, S.A., Allard, T., Jenkins, W.M. and Merzenich, M.M. (1988). Receptive fields in the body-surface map in adult cortex defined by temporally correlated inputs. Nature 332, 444445.

Cohen, N.J., Ryan, J., Hunt, C., Romine, L., Wszalek, T. and Nash, C. (1999). Hippocampal System and Declarative (Relational) Memory: Summarizing the Data From Functional Neuroimaging Studies. Hippocampus 9, 83-98

Corkin, S. (1968). Acquisition of motor skill after bilateral medial temporal-lobe excision. Neuropsychologia $6,225-264$.

Corkin, S, Amaral, D.G., Gonzalez, R.G., Johnson, K.A. and Hyman, B.T. (1997). H. M.'s Medial Temporal Lobe Lesion: Findings from Magnetic Resonance Imaging. Journal of Neuroscience 17, 3964-3979. 
Corkin, S. (2002). What's new with the amnesic patient H.M.? Nature Reviews Neuroscience 3 , 153-160.

Coward, L.A. (1990). Pattern Thinking, New York: Praeger.

Coward, L.A. (2000). A Functional Architecture Approach to Neural Systems. International Journal of Systems Research and Information Systems, 9, 69 - 120.

Coward, L.A. (2001). The Recommendation Architecture: lessons from the design of large scale electronic systems for cognitive science. Journal of Cognitive Systems Research 2(2), 111156.

Coward, L.A. (2004). Simulation of a Proposed Binding Model. Brain Inspired Cognitive Systems, L. S. Smith, A. Hussain and I. Aleksander, (editors), University of Stirling: Stirling.

Coward, L.A. (2005a). A System Architecture Approach to the Brain: from Neurons to Consciousness. New York: Nova Science Publishers.

Coward, L.A. (2005b). Accounting for episodic, semantic and procedural memory in the recommendation architecture cognitive model. Proceedings of the Ninth Neural Computation and Psychology Workshop: Modelling Language, Cognition, and Action.

Coward, L. A. and Gedeon, T.D. (2008). Implications of resource limitations for a conscious machine. Neurocomputing in press

Coward, L.A. and Gedeon, T.D. and Ratanayake, U. (2004). Managing Interference between Prior and Later learning. ICONIP 2004, Calcutta.

Coward, L. A. and Sun, R. (2007). Hierarchical Approaches to Understanding Consciousness. Neural Networks 20(9), 947 - 954

Curtis, C.E. and D'Esposito, M. (2003). Persistent activity in the prefrontal cortex during working memory. Trends in Cognitive Sciences 7, 415 - 423.

Dolcos, F., LaBar, K.S. and Cabeza, R. (2004). Interaction between the Amygdala and the Medial Temporal Lobe Memory System Predicts Better Memory for Emotional Events. Neuron $42,855-863$.

Eichenbaum, H., Otto, T. and Cohen, N.J. (1994). Two component functions of the hippocampal memory system. Behavioral and Brain Sciences 17, 449-517.

Euston, D.R., Tatsuno, M. and McNaughton, B.L. (2007). Fast-Forward Playback of Recent Memory Sequences in Prefrontal Cortex During Sleep. Science 318, 1147-1150.

Fosse, M.J., Fosse, R., Hobson, J.A. and Stickgold, R.J. (2003). Dreaming and Episodic Memory: A Functional Dissociation? Journal of Cognitive Neuroscience 15(1), 1 - 9.

Fyhn, M., Molden, S., Witter, M.P., Moser, E.I. and Moser, M-B. (2004). Spatial Representation in the Entorhinal Cortex. Science 305, 1258-1264.

Gedeon, T.D., Coward, L.A. and Bailing, Z. (1999). Results of Simulations of a System with the Recommendation Architecture, Proceedings of the 6th International Conference on Neural Information Processing, Volume I, 78-84.

Gilman, S., Bloedel J. R., and Lechtenberg, R. (1981). Disorders of the Cerebellum. Philadelphia, PA: FA Davis.

Gluck, M.A., Meeter, M. and Myers, C.E. (2003). Computational models of the hippocampal region: linking incremental learning and episodic memory. Trends in Cognitive Sciences 7(6), 269 - 276.

Goldman-Rakic, P. S. (1982). Cytoarchitectonic heterogeneity of the primate neostriatum: subdivision into island and matrix cellular compartments. Journal of Comparative Neurology 205, $398-413$

Graff-Radford, N.R., Tranel, D., Van Hoesen, G.W. and Brandt, J.P. (1990). Diencephalic Amnesia. Brain 113, 1 - 25.

Hassabis, D., Kumaran, D., Vann, S.D. and Maguire, E.A. (2007). Patients with hippocampal amnesia cannot imagine new experiences. Proceedings of the National Academy of Sciences (USA) 104(5), 1726 - 1731.

Hausser, M. and Mel, B. (2003). Dendrites: bug or feature? Current Opinion in Neurobiology 13, 372-383. 
Hoffman, K.L. and McNaughton, B.L. (2002). Sleep on it: cortical reorganization after the fact. Trends in Neuroscience 25(1), 1-2.

Hyvärinen, A., Karhunen, J. and Oja, E. (1999). Independent Component Analysis. New York: Wiley.

Insausti, R. and Amaral, D.G. (2004). Hippocampal Formation. In Paxinos G, Mai JK editors. The Human Nervous System. Elsevier. 871-914. .

Ishizuka, N., Weber, J. and Amaral, D.G. (1990). Organization of intrahippocampal projections originating from CA3 pyramidal cells in the rat. Journal of Comparative Neurology 295, 580 $-623$.

Kelley, A. E. (1999). Functional Specificity of Ventral Striatal Compartments in Appetitive Behaviors. Annals of the New York Academy of Sciences 877, 71-90.

Kesner, R.P., Lee, I. and Gilbert, P. (2004). A behavioral assessment of hippocampal function based on a subregional analysis. Reviews in the Neurosciences 15, 333-351.

Kesner, R.P. and Hopkins, R.O. (2006). Mnemonic functions of the hippocampus: A comparison between animals and humans. Biological Psychology 73, 3-18.

Kensinger, E.A., Ullman, M.T. and Corkin, S. (2001). Bilateral Medial Temporal Lobe Damage Does Not Affect Lexical or Grammatical Processing: Evidence From Amnesic Patient H.M. Hippocampus 11, 347-360.

Lavenex, P. and Amaral, D.G. (2000). Hippocampal-neocortical interaction: a hierarchy of associativity. Hippocampus $10,420-430$.

Lavenex, P., Suzuki, W.A. and Amaral, D.G. (2004). Perirhinal and Parahippocampal Cortices of the Macaque Monkey: Intrinsic Projections and Interconnections, Journal of Comparative Neurology 472, 371-394.

Leiner, H. C., Leiner, A. L. and Dow, R. S. (1993). Cognitive and language functions of the human cerebellum. Trends in Neuroscience 16, 444-447

Leutgeb, S., Leutgeb, J.K., Barnes, C.A., Moser, E.I., McNaughton, B.L. and Moser M-B. (2005). Independent codes for spatial and episodic memory in hippocampal neuronal ensembles. Science 309, 619-623.

Leutgeb, S., Leutgeb, J.K., Treves, A., Moser, M-B. and Moser, E.I. (2004). Distinct Ensemble Codes in Hippocampal Areas CA3 and CA1. Science 305, 1295-1298.

Lisman, J.E. (1999). Relating Hippocampal Circuitry to Function: Recall of Memory Sequences by Reciprocal Dentate-CA3 Interactions. Neuron 22, 233 - 242.

Lisman, J.E. and Otmakhova, N.A. (2001). Storage, Recall, and Novelty Detection of Sequences by the Hippocampus: Elaborating on the SOCRATIC Model to Account for Normal and Aberrant Effects of Dopamine. Hippocampus 11, 551-568.

Maguire, E.A., Gadian, D.G., Johnsrude, I.S., Good, C.D., Ashburner, J., Frackowiak, R.S.J. and Frith, C.D. (2000). Navigation-related structural changes in the hippocampi of taxi drivers. Proceedings of the National Academy of Sciences (USA) 97(8), 4398-4403.

McClelland, J.L., McNaughton, B.L. and O'Reilly, R.C. (1995). Why there are complementary learning systems in the hippocampus and neocortex: insights from the successes and failures of connectionist models of learning and memory. Psychological Review 102(3), 419-457.

McDonald, A. J. (1991). Organization of amygdaloid projections to the prefrontal cortex and associated striatum in the rat. Neuroscience 44(1), $1-14$.

Milner, B., Corkin, S. and Teuber, H-L. (1968). Further analysis of the hippocampal amnesic syndrome: 14-year follow-up study of H.M. Neuropsychologia 6, 215-234.

Mody, I. (2002). The GAD-given right of dentate gyrus granule cells to become GABAergic. Epilepsy Currents 2, 143-145.

Mountcastle, V.H. (2003). Introduction to special issue on cortical columns. Cerebral Cortex 13, 2-4.

Muller, W. and Misgeld, U. (1991). Picrotoxin- and 4-aminopyridine-induced activity in hilar neurons in the guinea pig hippocampal slice. Journal of Neurophysiology 65, 141-147. 
Naber, P.A., Witter, M.P. and Lopes da Silva, F.H. (1999). Perirhinal cortex input to the hippocampus in the rat: evidence for parallel pathways, both direct and indirect. A combined physiological and anatomical study. European Journal of Neuroscience 11, 4119-4133.

Nadel, L. and Moscovitch, M. (1997). Memory consolidation, retrograde amnesia and the hippocampal complex. Current Opinion in Neurobiology 7, 217-227.

Nauta, W. J. H., Smith, G. P., Faull, R. L. M. and Domesick, V. B. (1978) Neuroscience 3, 385401

Penttonen, M., Kamondi, K., Sik, A., Acsady, L. and Buzsaki, G. (1997). Feed-forward and feedback activation of the dentate gyrus in vivo during dentate spikes and sharp wave bursts. Hippocampus 7, 437-450.

Phelps, E.A. (2006). Emotion and Cognition: Insights from Studies of the Human Amygdala. Annual Review of Psychology 57, 27-53.

Ratnayake, U. and Gedeon, T.D. (2003). Extending The Recommendation Architecture Model for Text Mining. International Journal of Knowledge-Based Intelligent Engineering Systems, 7, 139-148.

Rekkas, P.V. and Todd Constable, R. (2005). Evidence That Autobiographic Memory Retrieval Does Not Become Independent of the Hippocampus: An fMRI Study Contrasting Very Recent with Remote Events. Journal of Cognitive Neuroscience 17, 1950-1961.

Rempel-Clower, N.L., Zola, S.M., Squire, L.S. and Amaral, D.G. (1996). Three Cases of Enduring Memory Impairment after Bilateral Damage Limited to the Hippocampal Formation. Journal of Neuroscience 16, 5233-5255

Rogers, T.T., Hocking, J., Noppeney, U., Mechelli, A., Gorno-Tempini, M.L., Patterson, K. and Price, C.J. (2006). Anterior temporal cortex and semantic memory: Reconciling findings from neuropsychology and functional imaging. Cognitive, Affective and Behavioral Neuroscience 6(3), $201-213$.

Sagar, J.H., Cohen, N.J., Corkin, S. and Growden, J.H. (1985). Dissociations among processes in remote memory. Annals of the New York Academy of Science 444, 533-535.

Schoenbaum, G. and Setlow, B. (2003). Lesions of Nucleus Accumbens Disrupt Learning about Aversive Outcomes. Journal of Neuroscience 23(30), 9833-9841.

Scoville, W.B. and Milner, B. (1957). Loss of recent memory after bilateral hippocampal lesions. Journal of Neurology, Neurosurgery, and Psychiatry 20, 11-21.

Sesack, S.R., and Carr, D.B. (2002). Selective prefrontal cortex inputs to dopamine cells: implications for schizophrenia. Physiology and Behavior 77, 513- 517.

Shibata, H. (1993). Direct Projections From the Anterior Thalamic Nuclei to the Retrohippocampal Region in the Rat. Journal of Comparative Neurology 337, 431-445.

Skaggs, W.E. and McNaughton, B.L. (1996). Replay of Neuronal Firing Sequences in Rat Hippocampus During Sleep Following Spatial Experience. Science 271, 1870 - 1873.

Skaggs, W.E,, McNaughton, B.L., Wilson, M.A. and Barnes, C.A. (1996). Theta phase precession in hippocampal neuronal populations and the compression of temporal sequences. Hippocampus $6,149-172$

Smith, M.L. (1988). Recall of Spatial Location by the Amnesic Patient H.M. Brain and Cognition 7, 178-183.

Squire, L.R. and Alvarez, P. (1995). Retrograde amnesia and memory consolidation: a neurobiological perspective. Current Opinion in Neurobiology 5, 169-177.

Suzuki, W.A. and Eichenbaum, H. (2000). The Neurophysiology of Memory. Annals of the New York Academy of Sciences 911, 175 - 191.

Suzuki, W. and Amaral, D.G. (1990). Cortical inputs to the CA1 field of the monkey hippocampus originates from the perirhinal and parahippocampal cortex but not from area TE. Neuroscience Letters 115, 43-48.

Suzuki, W.A. (1996). Neuroanatomy of the monkey entorhinal, perirhinal and parahippocampal cortices: Organization of cortical inputs and interconnections with amygdala and striatum. Seminars in Neurosciences 8, 3-12. 
Tamamaki, N., Abe, K. and Nojyo, Y. (1988). Three-dimensional analysis of the whole axonal arbors originating from single CA2 pyramidal neurons in the rat hippocampus with the aid of a computer graphic technique. Brain Research, 452, 255-272.

Tanaka, Y., Miyazawa, Y., Akaoka, F. and Yamada, T. (1997). Amnesia Following Damage to the Mammillary Bodies. Neurology 48, 160-165.

Tanaka, K. (2003). Columns for complex visual object features in the inferotemporal cortex: clustering of cells with similar but slightly different stimulus selectivities. Cerebral Cortex 13, 90-99.

Teyler, T.J. and DiScenna, P. (1986). The hippocampal memory indexing theory. Behavioral Neuroscience 100(2), 147 - 154

Thompson-Schill, S.L. (2003). Neuroimaging studies of semantic memory: Inferring "how" from "where." Neuropsychologia, 41, 280-292.

Tronson, N.C. and Taylor, J.R. (2007). Molecular mechanisms of memory reconsolidation. Nature Reviews Neuroscience 8, 262-275.

Tulving, E., Markowitsch, H.J., Craik, F.I.M., Habib, R. and Houle, S. (1996). Novelty and Familiarity Activations in PET Studies of Memory Encoding and Retrieval. Cerebral Cortex 6, 71-79.

Veazey, R.B., Amaral, D.G. and Cowan, W.M. (1982). The Morphology and Connections of the Posterior Hypothalamus in the Cynomolgus Monkey (Macaca fascicularis). 11. Efferent Connections. Journal of Comparative Neurology 207, 135-156.

Vertes, R.P. and Eastman, K.E. (2000). The case against memory consolidation in REM sleep. Behavioral and Brain Sciences 23, 867-876.

Walker, M.C., Ruiz, A. and Kullmann, D.M. (2001). Monosynaptic GABAergic signaling from dentate to CA3 with a pharmacological and physiological profile typical of mossy fiber synapses. Neuron 29, 703-715.

Wallenstein, G.V., Eichenbaum, H. and Hasselmo, M.E. (1998). The hippocampus as an associator of discontiguous events. Trends in Neuroscience 21, 317-323.

Wyss, J.M., Swanson, L.W. and Cowan, W.M. (1979). A study of subcortical afferents to the hippocampal formation in the rat. Neuroscience 4, 463-476.

Zola-Morgan, S., Squire, L.S. and Amaral, D.G. (1986). Human Amnesia and the Medial Temporal Region: Enduring Memory Impairment Following a Bilateral Lesion Limited to Field CA1 of the Hippocampus. Journal of Neuroscience 6(10), 2950-2967. 